\title{
A Numerical Study of Automotive Body Panel Draw Dies Defects Using Finite Element Simulation
}

\section{Farzad Yadegari ( $\nabla$ farzadyadegari@aut.ac.ir)}

Department of Mechanical Engineering, Amirkabir University of Technology (Tehran Polytechnic), Tehran, P.O.B. a15875-4413, Tehran, Iran

\section{Hossein Sehhat}

Department of Mechanical and Aerospace Engineering, Missouri University of Science and Technology, Rolla, MO 65409 https://orcid.org/0000-0002-7013-1642

\section{Ali Mahdianikhotbesara}

School of Mechanical Engineering, College of Engineering, University of Tehran, P.O. Box 11155/4563, Tehran, Iran https://orcid.org/0000-0002-9533-3259

\section{Research Article}

Keywords: Simulation, Finite Element Method, Optimization, Car Body, Sheet Metal Forming

Posted Date: February 2nd, 2022

DOI: https://doi.org/10.21203/rs.3.rs-1300589/v2

License: (c) (i) This work is licensed under a Creative Commons Attribution 4.0 International License. Read Full License 


\title{
A Numerical Study of Automotive Body Panel Draw Dies Defects Using Finite Element Simulation
}

\author{
Farzad Yadegari ${ }^{1, a^{*}}$, M. Hossein Sehhat ${ }^{2, b}$, Ali Mahdianikhotbesara ${ }^{3, c}$ \\ ${ }^{1}$ Department of Mechanical Engineering, Amirkabir University of Technology (Tehran Polytechnic), \\ Tehran, P.O.B. a15875-4413, Tehran, Iran \\ ${ }^{2}$ Department of Mechanical and Aerospace Engineering, Missouri University of Science and Technology, \\ Rolla, MO 65409 \\ ${ }^{3}$ School of Mechanical Engineering, College of Engineering, University of Tehran, P.O. Box 11155/4563, \\ Tehran, Iran \\ a* $\underline{\text { aarzadyadegari@aut.ac.ir }}, \underline{\text { bhsehhat@mst.edu }}, \underline{\text { calimahdiani@ut.ac.ir }}$
}

\begin{abstract}
Among the advantages of the sheets used in the car body, it can mention the high strength, low thickness and suitable ductility. crack and wrinkling of the sheet are the most common types of defects in the drawing process. Therefore, it seems necessary to know the process factors that affect the sheet metal forming behavior. One of the critical tools of simulation and subsequent process optimization is the finite element method. In this research, with the help of AutoForm software, finite element simulation of the process is performed. After designing the appropriate Taguchi experiment, the parameters affecting the outputs, including thickness strain and surface strain on the production part, are investigated, and optimal values are introduced. The known effective parameters on the process output include the forming limit diagram, sheet strength, friction coefficient, die balancers, and used sheet thickness.
\end{abstract}

Keywords: Simulation, Finite Element Method, Optimization, Car Body, Sheet Metal Forming

\section{Introduction}

Today, in different industries, new methods are used to produce products [1]-[5]. The use of these new technologies, such as lasers [6]-[8] and some other older methods such [9]-[11] can improve the performance of some of these products. One of the industries that currently makes much use of parts produced by metal dies is the automotive industry. Other industries such as others, oil, transportation, etc., can also be considered consuming these dies. Press dies are among the metal dies by which metal sheets are formed. Advances in automotive technology, improving the quality of products offered in global markets, and increasing the level of production expectations of industrial products, made new quality production methods more limited and common, in addition to maintaining the quality product line of production units of applications. From an engineering point of view, the functionality and suitability of the product before mass production should be examined in bulk, and the most appropriate material and production method should be selected under specified conditions and take into account manufacturing costs. The finite analysis is the most common method used. An engineer can check if the product is working well by performing 
finite element analysis before making a fundamental part. If the product becomes problematic in the analytical method, the issues related to the subject can be redefined, or the material can be replaced. Therefore, before starting construction in the workshop, it is essential to perform a wellplanned and appropriate analysis to determine which production method is more suited to build a product with the desired quality and the lowest manufacturing cost. This is where R\&D units in factories come into play [12]-[15].

\section{Literature review}

Numerous researchers have used analytical and theoretical methods to predict the phenomena of thinning and rupture in the process of deep tension. In most of these studies, by using modeling of a cup tensile test (due to the ease of symmetrical axial geometry analysis) and its theoretical analysis in finite element software and sometimes die construction to match experimental and theoretical results, the sheet behavior is investigated. The scope of application of this method is in designing and manufacturing the mechanism of metal dies of the car body and producing pressed parts. Changing the behavior of the sheet in this process is desired, and examining and analyzing it leads to design and manufacturing principles with less error. In dieing companies, this method can be used in all stages before and after designing stretch dies. It is worth mentioning that in most large dieing companies globally, this method is used as a complementary experiment and simulation software. Another common point that can be seen in these articles is the discussion of experimental design in them so that after the initial experiments on the desired process and identification of parameters affecting its results, based on the need and type of purpose of the research. For example, in the papers, the three parameters of binder force, edge radius, and coefficient of friction in the deep tensile test of a steel sheet based on the design of the Taguchi experiment have been studied, and the parameters have been optimized [16]-[19].

\section{Study model}

In this study, to determine the behavior of DC04 tensile steel sheet with standard number EN10130, which is one of the most widely used sheets in car bodies, in case of rupture and thinning defects, it has been studied on the draw die of the front door of Peugeot 405 car. For this purpose, first, the die simulation was performed in AutoForm software. After determining the critical and high-risk areas, the Taguchi method designed the experiment to achieve optimal values for process parameters [20]. The illustration of this die is shown in table1). 


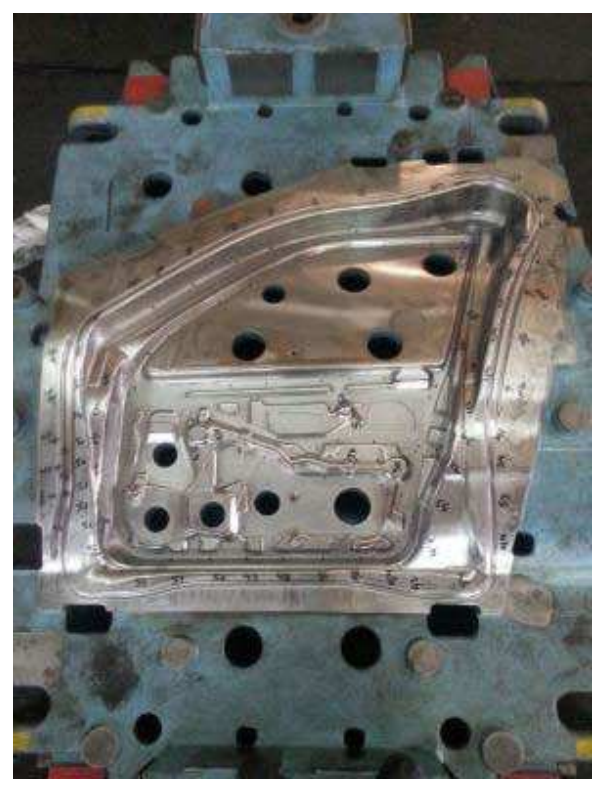

Fig. 1 Case study: Peugeot 405 front door draw die

\section{Simulation of the die using software}

The model studied in this simulation first achieved the desired geometry by making modifications in CATIA software to optimize the process. These changes include the elimination of discontinuities and surface differences between different parts of the model, etc., which have been done to bring the model levels closer to the primary levels of the die. The process was then simulated using AutoForm software. The geometry of the studied model is shown in Figure (2).

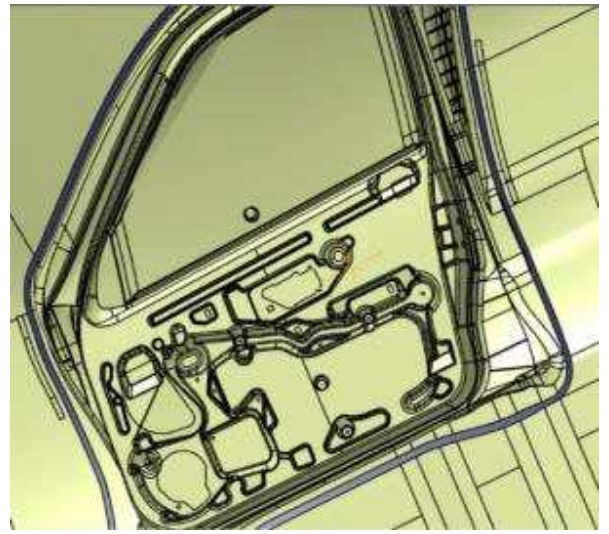

Fig. 2 Die geometry in CATIA software

\section{Simulation steps}

First, by entering the geometry in AutoForm software, the best direction for the drawing process was selected so that the minor areas are prone to problems such as the panel coming out of the die (negative slope), rupture, etc. The software categorizes geometry into three levels and presents it to the designer in Figure (3). In this figure, as can be seen, the yellow areas are the zones where 
the mentioned problems are likely to occur. The possibility is that it can be optimally reduced by making conscious changes to the process parameters and optimizing them. While green areas do not generally cause problems, some process parameters have not yet been introduced to the software at this stage, parameters such as press force, friction, etc., which are very important in the quality of the final part.

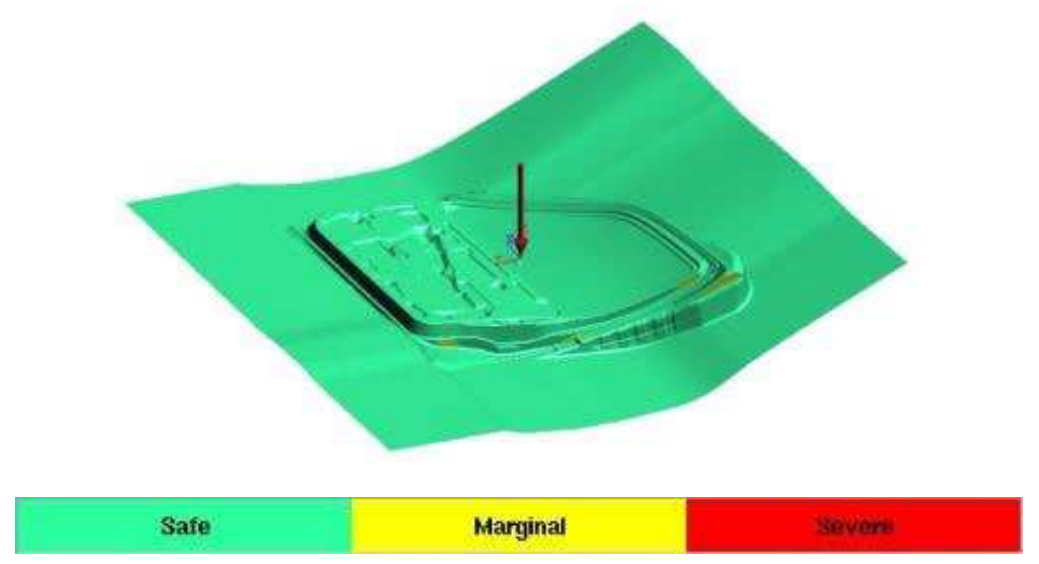

Fig. 3 Software prediction of critical areas of the part

After finding the best direction of the pressing process, it is time to define the inputs and other process parameters. This step is done using the Process Generator icon. The first tab of this window is related to the dimensions, position, material, and properties of the sheet used. The blank created and other sheet specifications are given in Figures (4) and (5), respectively.

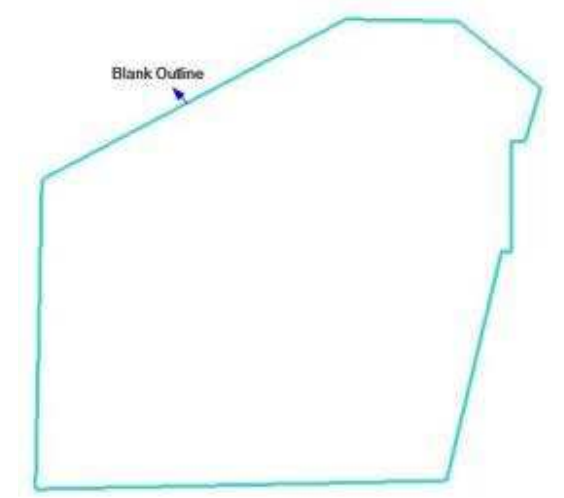

Fig. 4 Blanks created in the software 


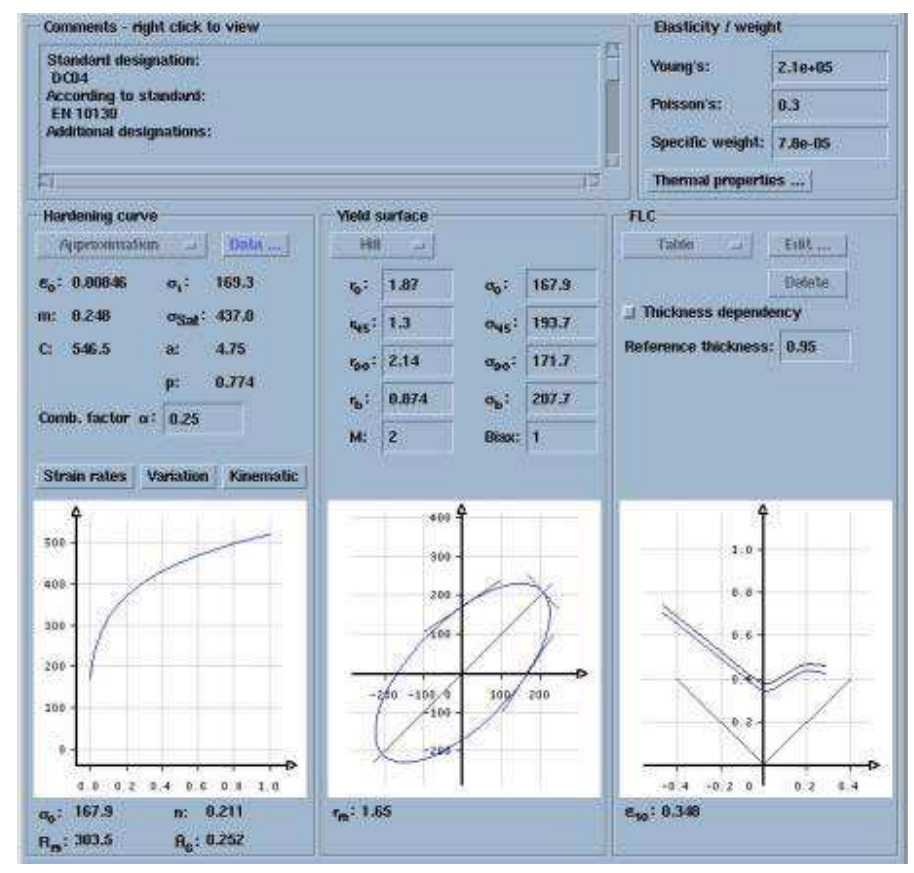

Fig. 5 Specifications of the sheet used in the simulation process (taken from the software database)

Now it is time to define the die components. This is done in the Tools Tab. Depending on the type of press used, which is a single-action type, these components, including the punch, die, and binder, are defined as shown in Figure (6).

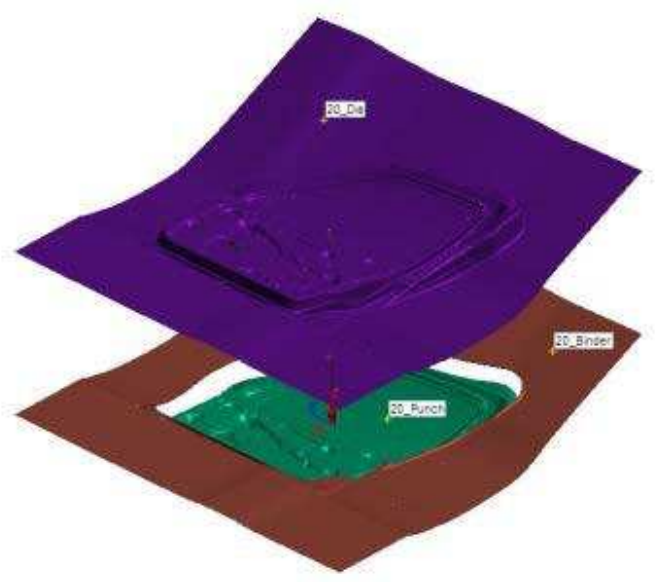

Fig. 6 Introduce the main components of the die to the software

\section{Draw bead simulation}

Draw beads are one of the die components that are machined in the form of protrusions and indentations on the punch and binder. The function of the beads is to control the rate of tension of the sheet and the flow of the material, as well as the applied stresses on the sheet. The simulation performed is shown in Figure (7). 
As shown in the figure, in the present model, and all-around draw bead and three cross-section draw beads are used in front, rear, and above the car door.

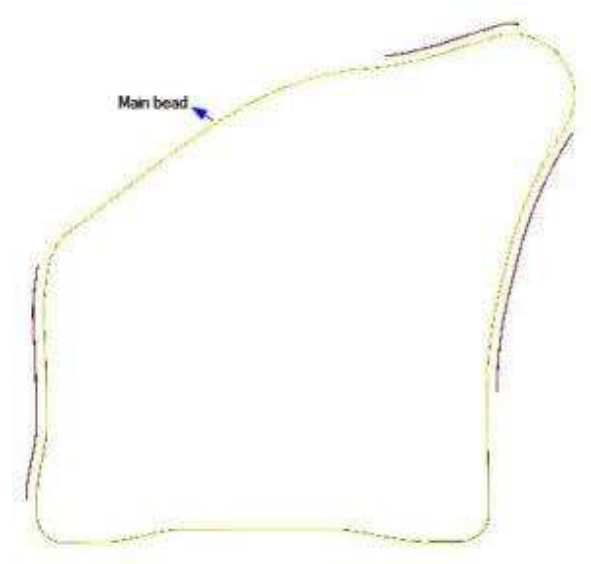

Fig. 7 Simulated draw beads in software

The draw bead has different widths at different distances. These areas and the width of each of them are shown in Figure (8). It should be noted that these differences in size are due to the asymmetric geometry and its different complexities to control the flow of the sheet and its flexibility.

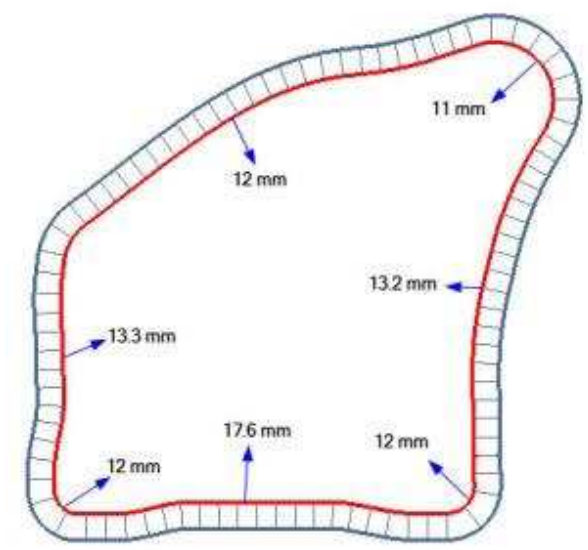

Fig. 8 Different widths of draw bead at different distances

\section{Balancer simulation}

Spacers or balancers are spacing blocks used to control the sheet's movement to move the sheet's tension and flow locally at different points. When the sheet comes down, it stops at a certain point and stops coming down. The sheet holder has a definite course, and in order not to increase the course, blocks are installed on the punch and the sheet holder so that when the sheet holder reaches the end of its course, these blocks also come together, and the sheet holder does not come down. St37 or MS60 is sometimes used for the material of these blocks. The height of the spacers is 
adjusted by placing thin metal plates called lathes under them. Figures (9) and (10) show pictures of the spacers used in the die.

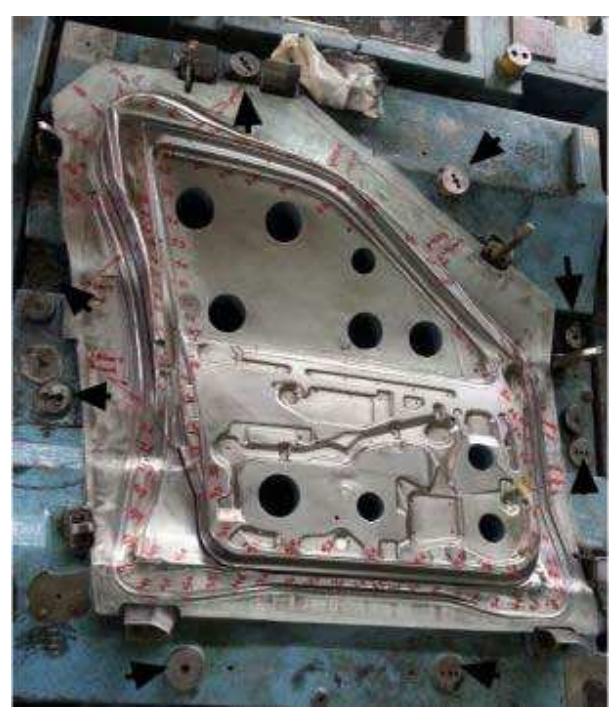

Fig. 9 View of the spacers used in the study format

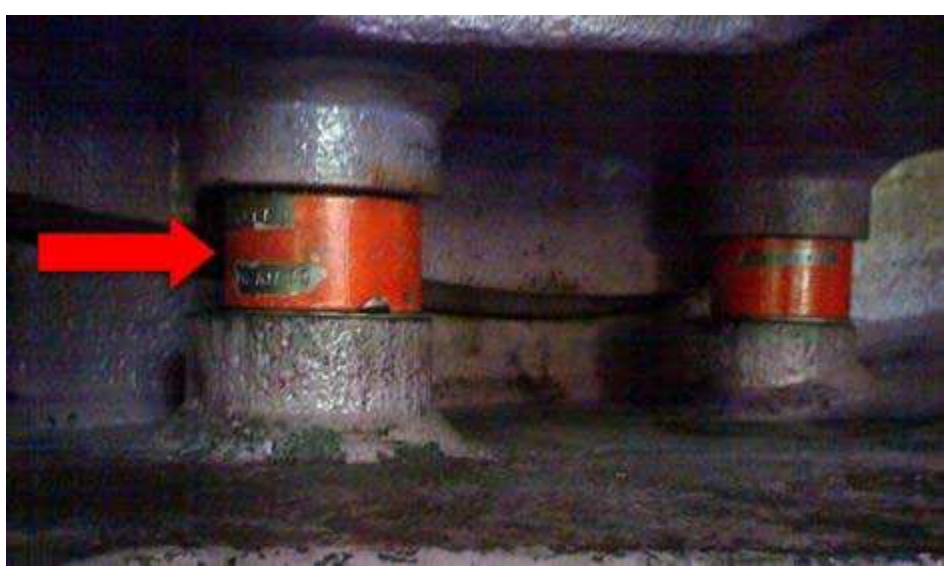

Fig. 10 Spacers used in the die

In Figure (11), the simulated position of the spacers can be seen in the studied die model. 


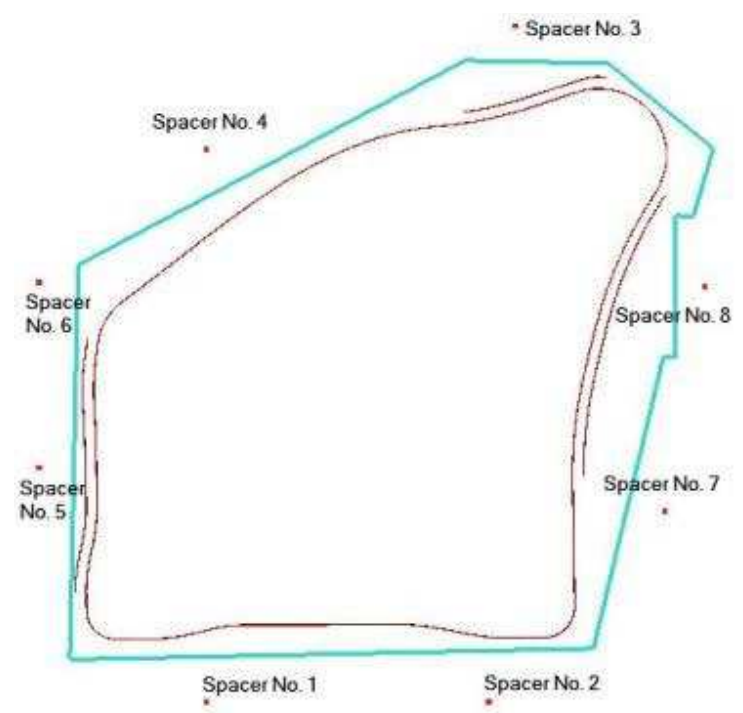

Fig. 11 Position of simulated spacers in the software

Due to the higher tensile depth and complexity of the die geometry at the top and rear of the car door will result in higher stresses on the part, the balancers in these areas (balancers 3, 4, 7, and 8) are set at a higher height.

\section{Air pin simulation}

Sheet force plays an essential role in the formability and flow rate of the sheet in draw dies. The transfer of this force to the sheet is the responsibility of the air pins. The right choices in this zone can prevent the sheet from tearing or shrinking. The image of the air pin and the simulation performed in Figures (12) and (13) can be seen.

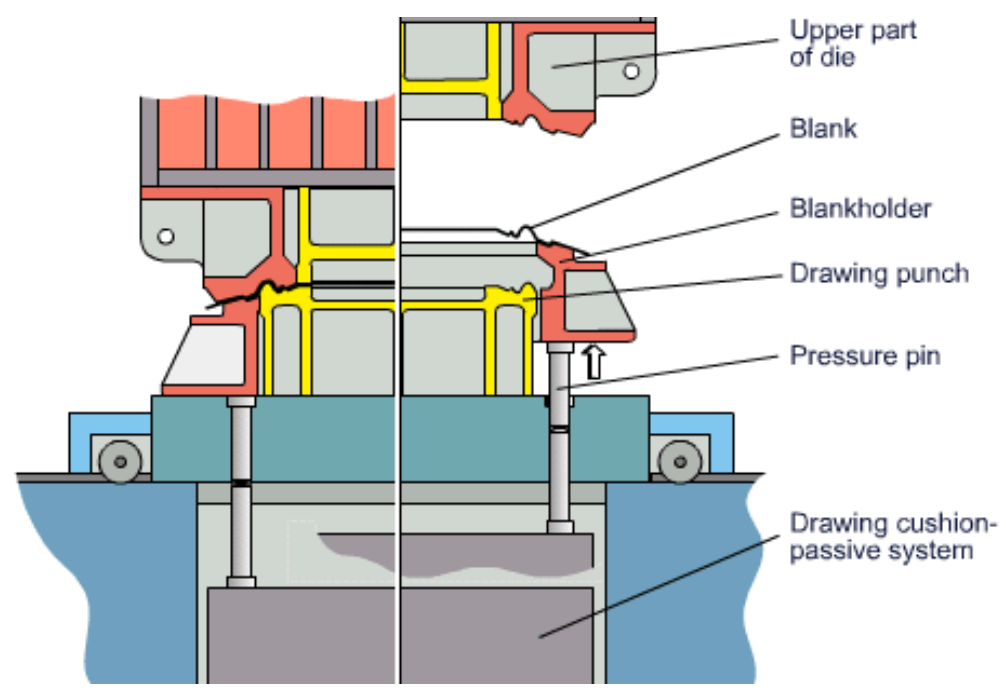

Fig. 12 The position and function of the air pin in the die [21] 


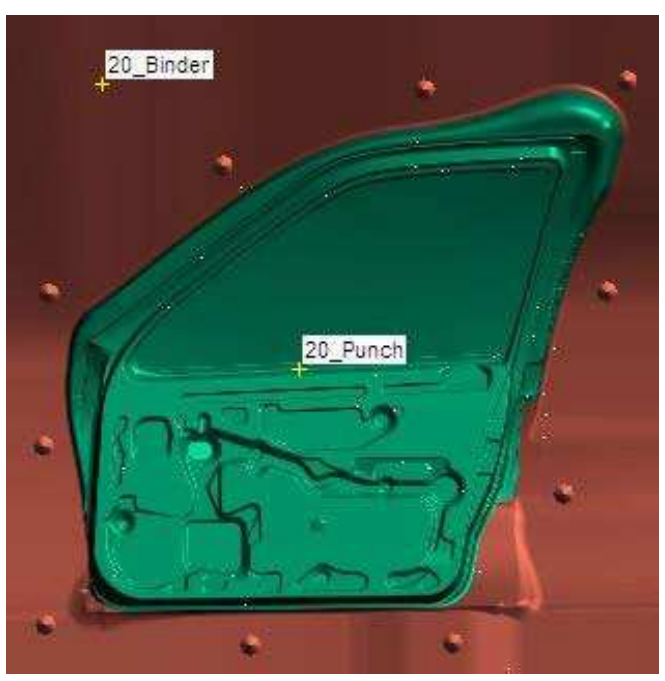

Fig. 13 Simulated position of air pins

\section{Other simulation parameters}

By selecting standard lubrication on the surfaces of the die and the punch, the coefficient of friction was determined to be 0.15 . The holder force is also set at 70 tons.

According to the factory settings, the total process time is 4 seconds, which will be done in the first 2 seconds of the sheeting operation and in the last 2 seconds of the drawing operation. According to the factory settings and according to the time intervals of the process, the length of the sheet and punch motion course is considered to be 170 and $1050 \mathrm{~mm}$, respectively. Thus, the speed up to the time of punch and binder engagement will be equal to $440 \mathrm{~mm} / \mathrm{s}$, and at the time of drawing will be equal to $85 \mathrm{~mm} / \mathrm{s}$.

\section{Formability of simulated model}

In Figures (14) to (17), the formability stages of the part at different times are shown.

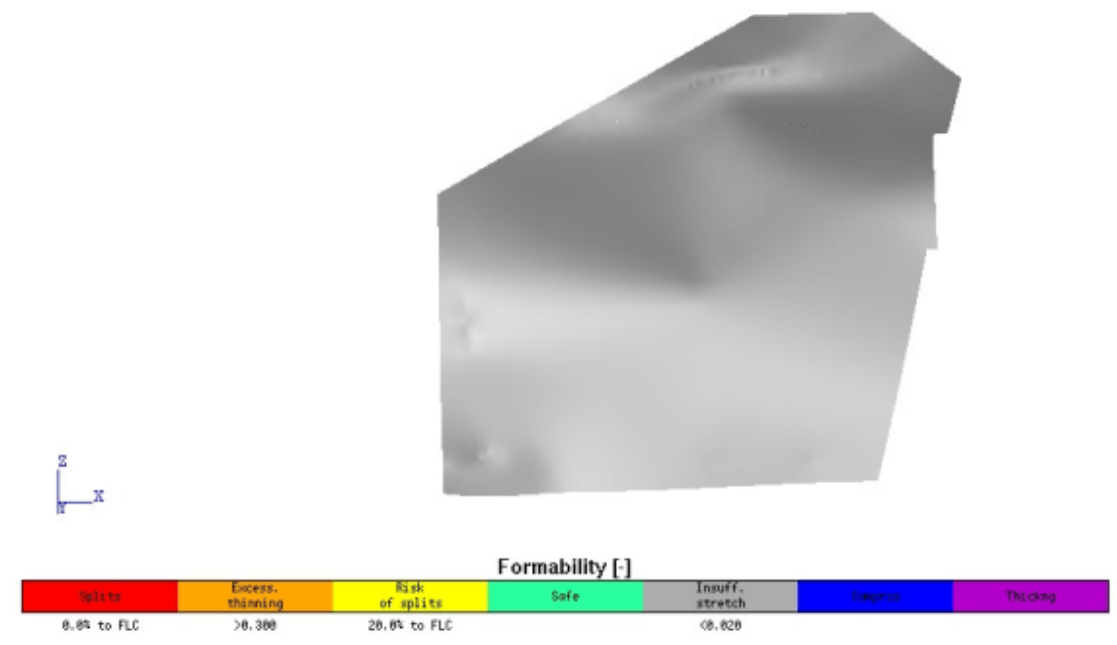

Fig. 14 the form created on the sheet in 2 seconds 


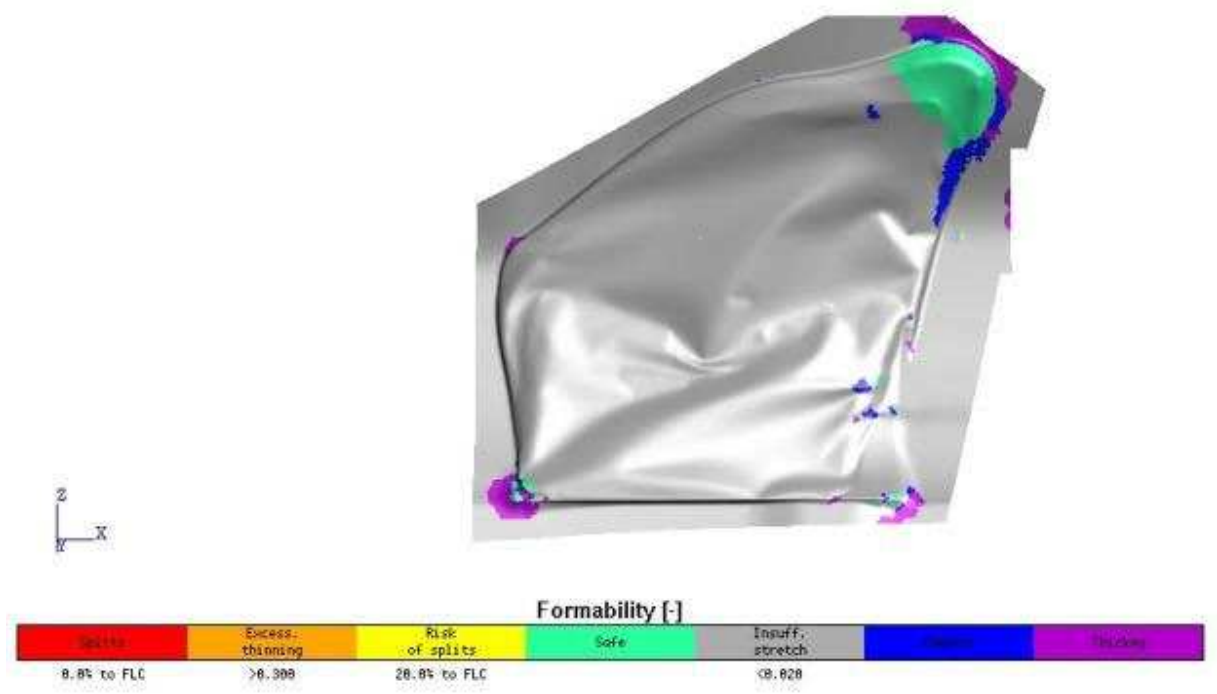

Fig. 15 the form created on the sheet in 3 seconds

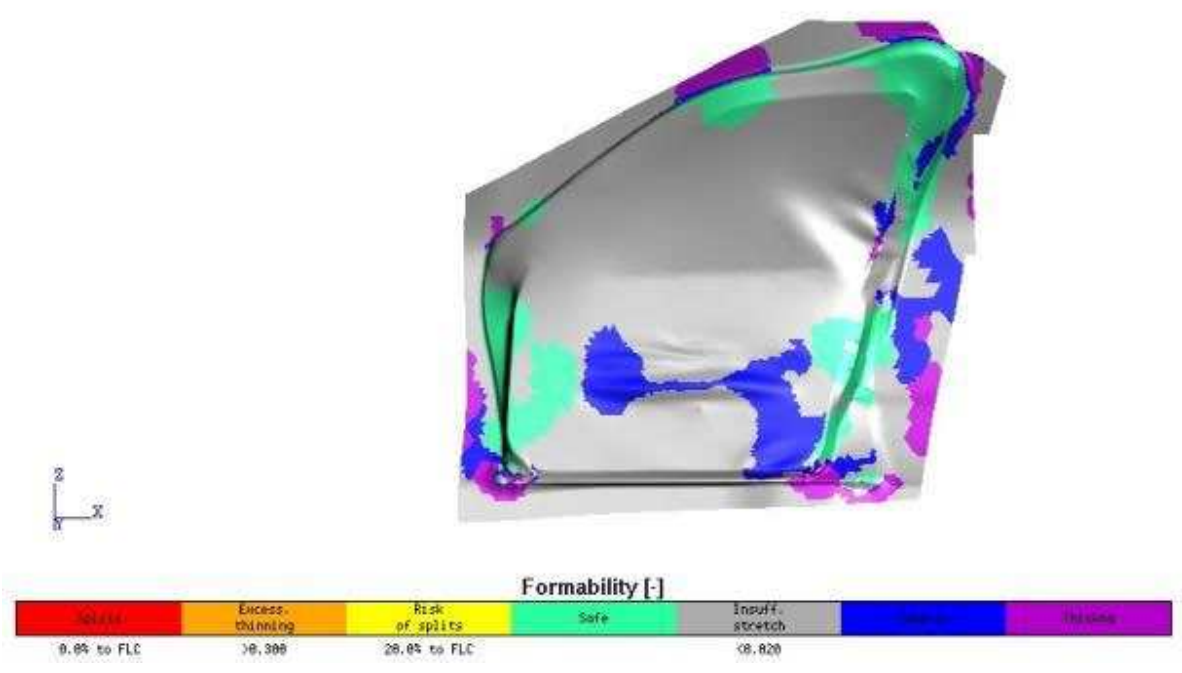

Fig. 16 the form created on the sheet in 3.5 seconds 


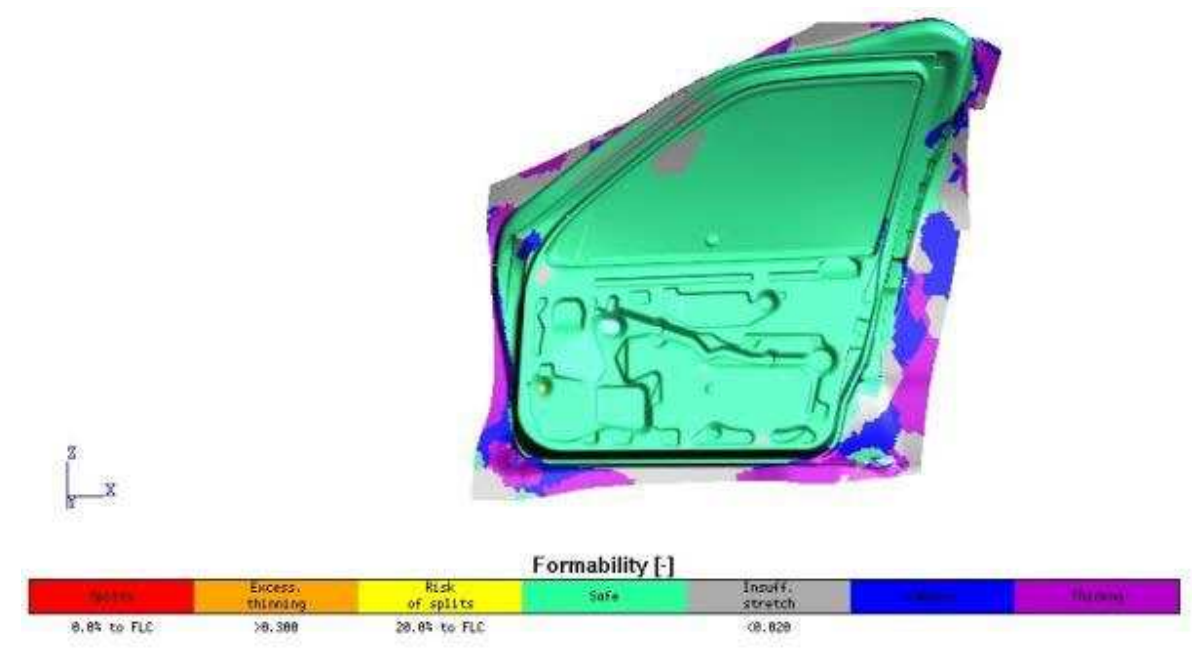

Fig. 17 the form created on the sheet in 4 seconds (end of the process)

The forces applied to the die components during the process over time can be seen in Figure (18).

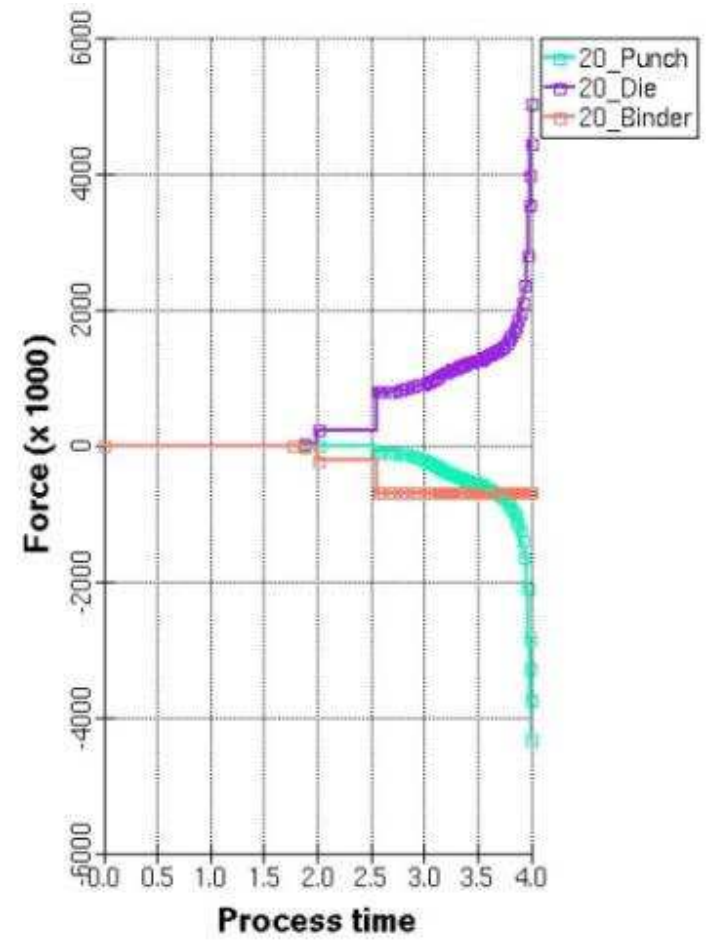

Fig. 18 Forces applied to die components during the process (Newton)

Figure (19) shows the forming limit diagram of the part at the end of the process. This diagram shows the location of critical defects on the sheet and helps estimate the occurrence of defects in these areas. As can be seen in this figure, red indicates the rupture range, green indicates the safe range, purple indicates the thickening range, yellow indicates the risk of rupture, and blue indicates the compaction of the material. Black dots are the maximum and minimum strain values of the simulated part elements, which can be seen in areas where the part is thinned or torn. 


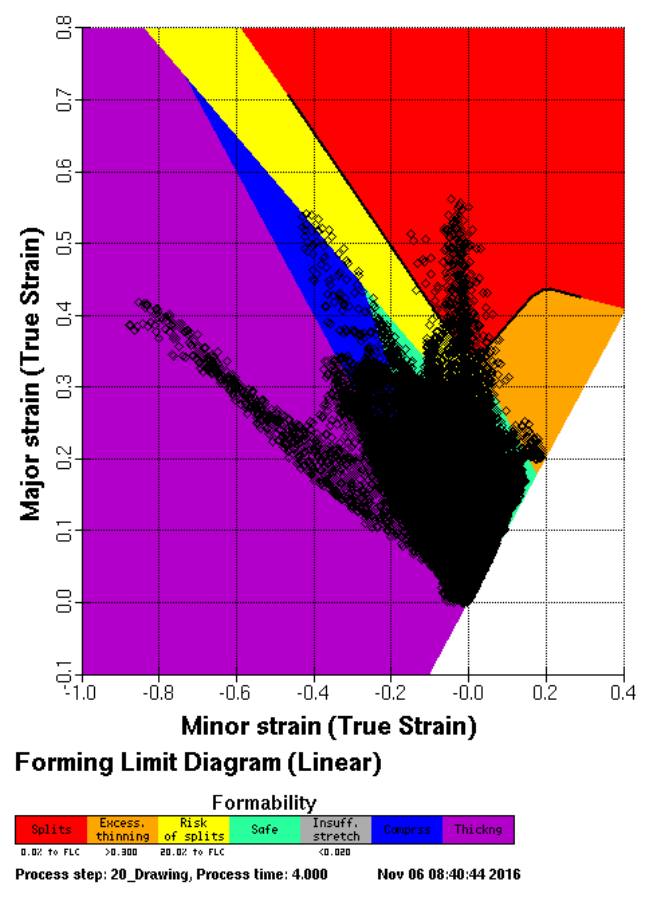

Fig. 19 FLD diagram based on formability

\section{Experimental design}

After the simulation is done, it is time to identify and determine the critical zones. In order to optimize the simulation parameters, four critical regions were selected using formability results. For this reason, four zones have been studied that the type of geometry and its complexity, the depth of draw and the nature of the tool engagement with the sheet, the height of the balancers used, the shape and size of the machined mounds in each of these zones are different. have been. It is worth mentioning that these zones have been selected in such a way that by making corrections and improving the formability in them, the formability of other surrounding zones will also be enhanced with a high percentage of confidence. The approximate position of each of these zones is given in Figure (20), and the detailed position along with their formability results is given in Figures (21) to (24). 


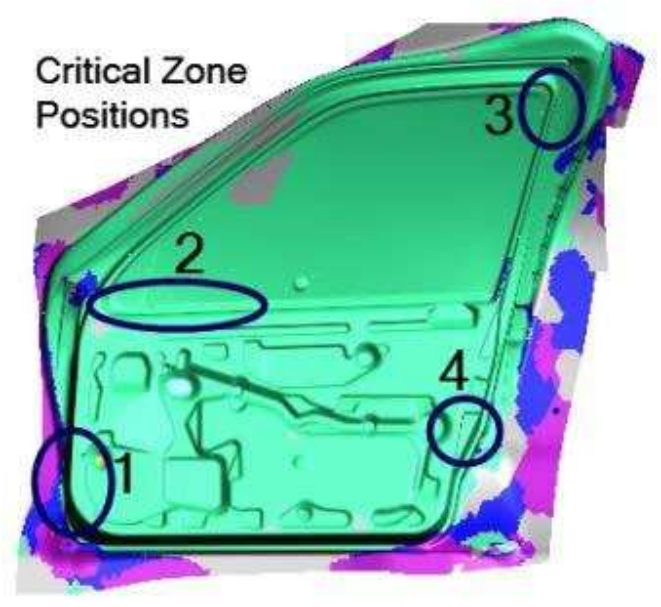

Fig. 20 Approximate location of designated critical zones

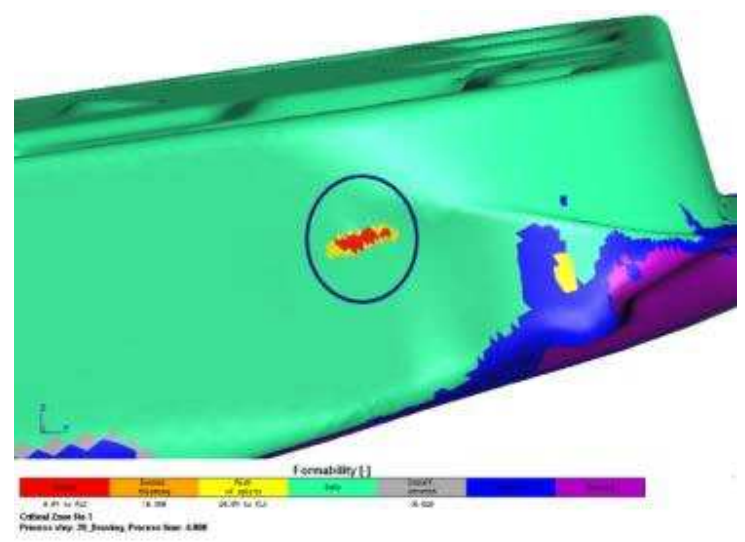

Fig. 21 Critical Zone No. 1

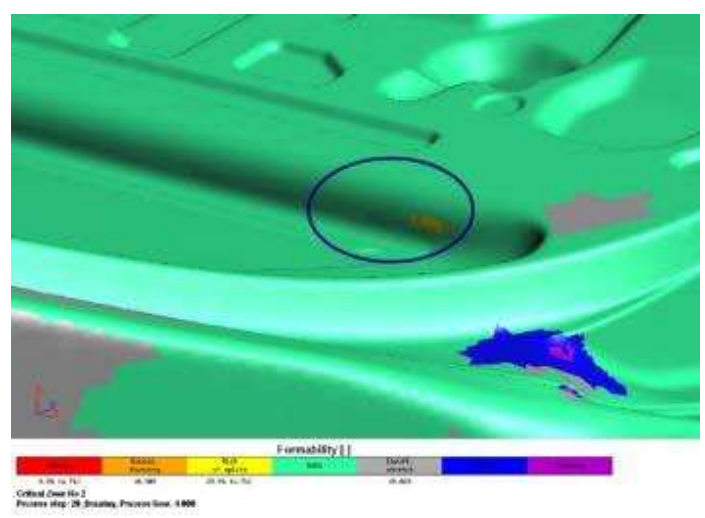

Fig. 22 Critical Zone No. 2 


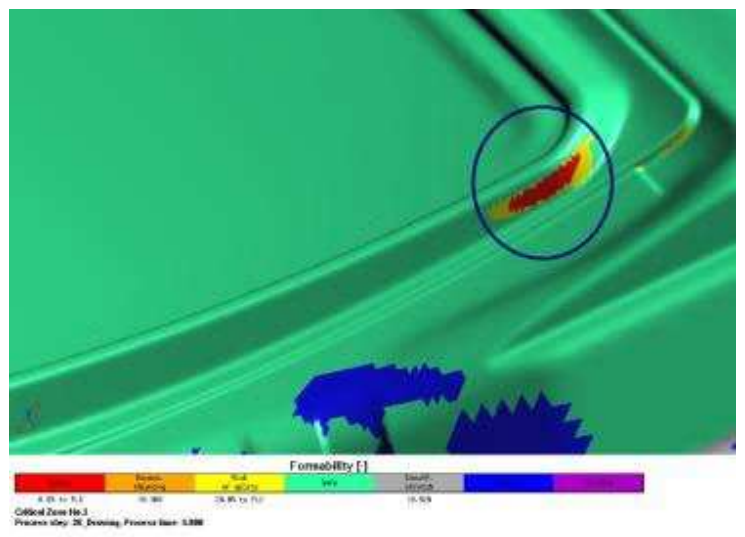

Fig. 23 Critical Zone No. 3

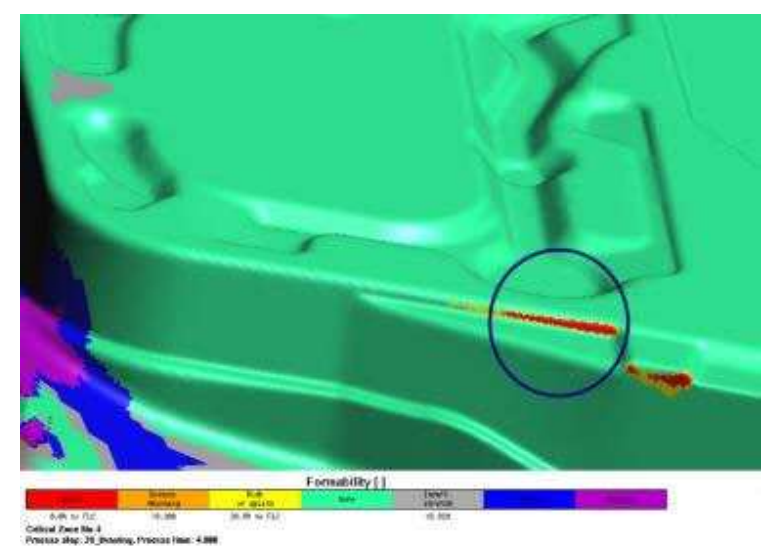

Fig. 24 Critical Zone No. 4

\section{Design of experiment}

After selecting the critical zones, a suitable experiment can be designed and performed so that the optimal levels of the parameters can be achieved by examining the results. But before designing the experiment, we have another review of the values of the simulated model parameters so that they can be used to determine the levels to be tested.

- Sheet material used: DC04 with properties and forming limit diagram in Figure (5)

- Binder force: 70 tons

- Friction coefficient: 0.15

- Relative height of spacers $1,2,5$, and 6: equal to 1.3 and spacers 3,4 , 7, and 8: equal to $1.8 \mathrm{~mm}$

- Sheet thickness used: $0.7 \mathrm{~mm}$ 


\section{Description of the test method}

Due to the fierce competition between industries, high-quality products and low prices are the keys to success and stability in the international arena. For this reason, in the research and development centers of factories, a lot of money is spent on optimizing the production process through experiments. Since such experiments are time-consuming and costly due to the diversity of production parameters and their interaction, the use of different methods in the design of these experiments can reduce the number of experiments. It is necessary. Taguchi experimental design is one of the methods used in experimental design, the implementation of which has been associated with many successes in various industries. The Taguchi method is quite different from the conventional methods of quality engineering. Taguchi methodology emphasizes quality design during product and process design, while traditional methods are based on quality inspection and control during or after production. Taguchi has used quite common statistical tools in his methods of increasing quality. But he has simplified these methods by identifying robust solutions in designing experiments and analyzing results. The Taguchi method has been very effective in increasing the quality of Japanese products. Recently, Western industrialized countries have also used the Taguchi method as a simple and effective method to improve outcomes and increase process quality in their industries. The method of designing Taguchi experiments has wide applications in various industries. An essential feature in the analysis of Taguchi test results is SNR diagrams, which reduce their impact by identifying uncontrollable factors that create noise in the results, according to the user's demands on the software. In these diagrams, considering that the effect of all parameters on the outputs under study is measured with a so-called weight and scale, it is possible to understand the impact of each parameter. In this case, changes can be made with more focus on parameters that have a more prominent role in the process, and, of course, more accurate results can be achieved at a lower cost. For this purpose, after modeling the studied geometry, the desired parameters are classified into three levels Table (1) and 27 experiments software as seen in Table (2), was designed.

Table 1 Parameters and levels of experiments

\begin{tabular}{c|ccccc} 
& FLD boundries & $\begin{array}{c}\text { Holder force } \\
\text { (ton) }\end{array}$ & $\begin{array}{c}\text { Friction } \\
\text { coefficeint }\end{array}$ & $\begin{array}{c}\text { Spacer (relative } \\
\text { height) }\end{array}$ & $\begin{array}{c}\text { Sheet thickness } \\
\text { (mm) }\end{array}$ \\
\hline 1 & $15 \% \downarrow$ & $15 \% \downarrow$ & $0 / 1$ & $15 \% \downarrow$ & 0.6 \\
2 & Normal & Normal & $0 / 15$ & Normal & 0.7 \\
3 & $15 \% \uparrow$ & $15 \% \uparrow$ & $0 / 17$ & $15 \% \uparrow$ & 0.8
\end{tabular}


Table 2 Values of experiments by Taguchi

\begin{tabular}{|c|c|c|c|c|c|}
\hline Parameters & FLD boundries & $\begin{array}{l}\text { Holder force } \\
\text { (ton) }\end{array}$ & Friction coefficeint & $\begin{array}{c}\text { Spacer (relative } \\
\text { height) }\end{array}$ & $\begin{array}{c}\text { Sheet } \\
\text { thickness } \\
\text { (mm) }\end{array}$ \\
\hline 1 & $15 \% \downarrow$ & $15 \% \downarrow$ & $0 / 1$ & $15 \% \downarrow$ & 0.6 \\
\hline 2 & $15 \% \downarrow$ & $15 \% \downarrow$ & $0 / 1$ & $15 \% \downarrow$ & 0.7 \\
\hline 3 & $15 \% \downarrow$ & $15 \% \downarrow$ & $0 / 1$ & $15 \% \downarrow$ & 0.8 \\
\hline 4 & $15 \% \downarrow$ & Normal & $0 / 15$ & Normal & 0.6 \\
\hline 5 & $15 \% \downarrow$ & Normal & $0 / 15$ & Normal & 0.7 \\
\hline 6 & $15 \% \downarrow$ & Normal & $0 / 15$ & Normal & 0.8 \\
\hline 7 & $15 \% \downarrow$ & $15 \% \uparrow$ & 0/ 17 & $15 \% \uparrow$ & 0.6 \\
\hline 8 & $15 \% \downarrow$ & $15 \% \uparrow$ & 0/ 17 & $15 \% \uparrow$ & 0.7 \\
\hline 9 & $15 \% \downarrow$ & $15 \% \uparrow$ & $0 / 17$ & $15 \% \uparrow$ & 0.8 \\
\hline 10 & Normal & $15 \% \downarrow$ & $0 / 15$ & $15 \% \uparrow$ & 0.6 \\
\hline 11 & Normal & $15 \% \downarrow$ & 0/ 15 & $15 \% \uparrow$ & 0.7 \\
\hline 12 & Normal & $15 \% \downarrow$ & $0 / 15$ & $15 \% \uparrow$ & 0.8 \\
\hline 13 & Normal & Normal & $0 / 17$ & $15 \% \downarrow$ & 0.6 \\
\hline 14 & Normal & Normal & 0/ 17 & $15 \% \downarrow$ & 0.7 \\
\hline 15 & Normal & Normal & $0 / 17$ & $15 \% \downarrow$ & 0.8 \\
\hline 16 & Normal & $15 \% \uparrow$ & $0 / 1$ & Normal & 0.6 \\
\hline 17 & Normal & $15 \% \uparrow$ & $0 / 1$ & Normal & 0.7 \\
\hline 18 & Normal & $15 \% \uparrow$ & $0 / 1$ & Normal & 0.8 \\
\hline 19 & $15 \% \uparrow$ & $15 \% \downarrow$ & 0/ 17 & Normal & 0.6 \\
\hline 20 & $15 \% \uparrow$ & $15 \% \downarrow$ & 0/ 17 & Normal & 0.7 \\
\hline 21 & $15 \% \uparrow$ & $15 \% \downarrow$ & 0/ 17 & Normal & 0.8 \\
\hline 22 & $15 \% \uparrow$ & Normal & 0/ 1 & $15 \% \uparrow$ & 0.6 \\
\hline 23 & $15 \% \uparrow$ & Normal & 0/1 & $15 \% \uparrow$ & 0.7 \\
\hline 24 & $15 \% \uparrow$ & Normal & $0 / 1$ & $15 \% \uparrow$ & 0.8 \\
\hline
\end{tabular}




\begin{tabular}{|l|l|l|r|r|}
\hline 25 & $15 \% \uparrow$ & $15 \% \uparrow$ & $0 / 15$ & $15 \% \downarrow$ \\
\hline 26 & $15 \% \uparrow$ & $15 \% \uparrow$ & $0 / 15$ & $15 \% \downarrow$ \\
\hline 27 & $15 \% \uparrow$ & $15 \% \uparrow$ & $0 / 15$ & $15 \% \downarrow$ \\
\hline
\end{tabular}

\section{Results and their interpretation}

This section interprets and analyzes the results of the experiments performed. These results are presented in two outputs of "thinning" and "main strains" on the four critical areas. How to distribute the thickness in different parts of the panel surfaces and the surface strains at the end of the tensile process for the simulation performed is shown qualitatively in Figures (25) to (27). Figure (25) shows that the zones that were previously identified by the software as prone to rupture have the highest rate of thinning. This is also evident in the way the principal strains are distributed.

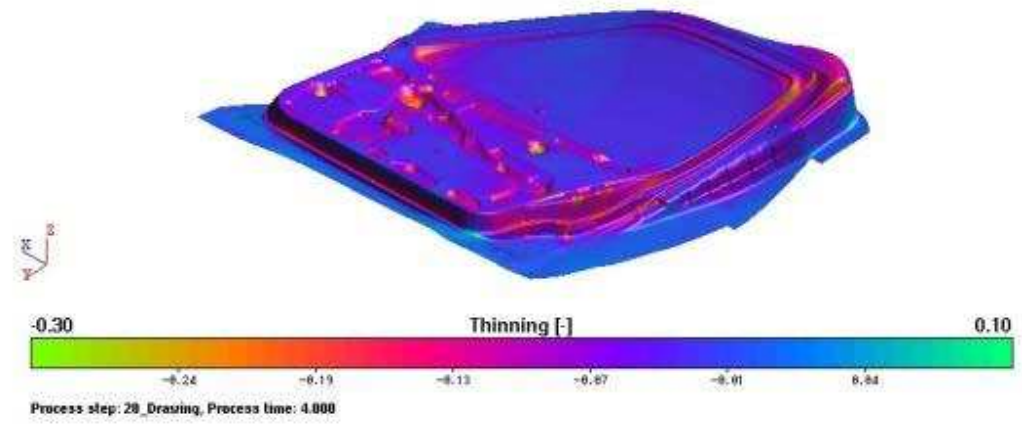

Fig. 25 Distribution of the thickness

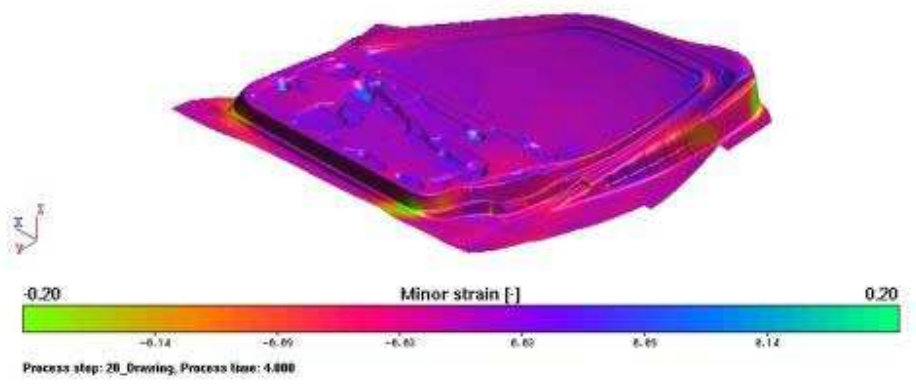

Fig. 26 Distribution of the minor strain 


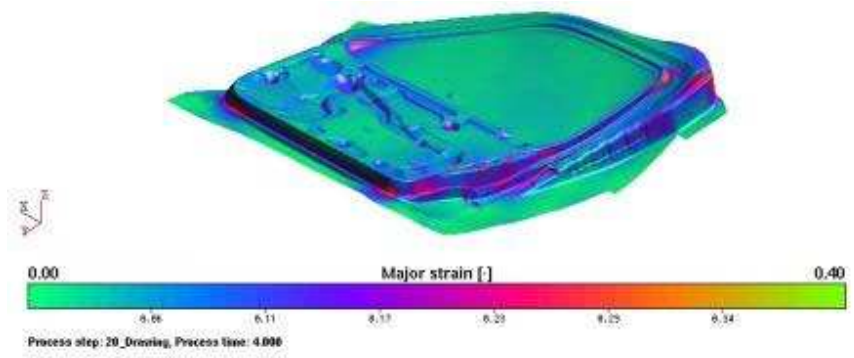

Fig. 27 Distribution of the maximum strain

In Figure (28), which shows the distribution of minimum and maximum principal strains, light blue dots representing the simulation elements and dark blue dots representing the distribution of strains in the simulation are performed.

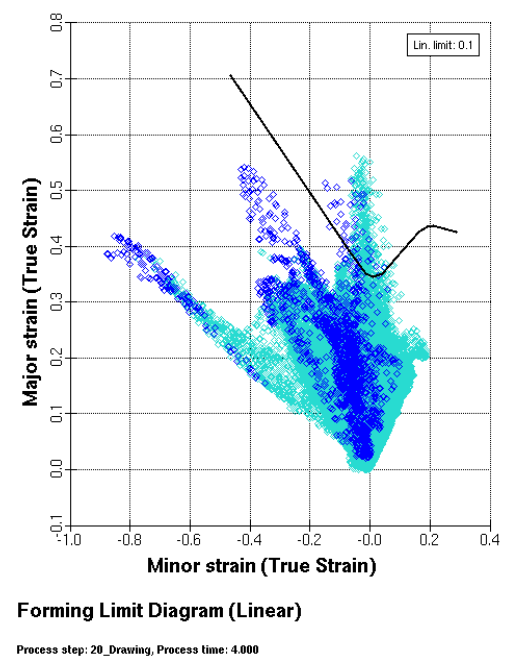

Fig. 28 Process FLD diagram based on strain distribution

\section{Investigating the effect of parameters on thinning}

In the SNR analysis, the lower percentage of thinning and the lower risk of rupture of the sheet at a given shaping rate means the better process is performed "smaller is better" mode is selected. The result of SNR analysis of the Taguchi experiment in the specified condition showed the dominant effect of changes in the parameters of thickness, coefficient of friction, and forming limit diagram. This is shown as an example for critical zone 1 in Figure (29). In the horizontal axis of this diagram, the levels of each parameter and in its vertical column, the SNR value are shown.

It should be noted that in this type of analysis, the higher the SNR value at one level, it means that the test results at that level are closer to the set goal. For example, it can be said that in coefficients of $0.1,0.15$, and 0.17 , the approximate SNR values of 14.5, 14.1, and 13.5, respectively, are obtained, which in the coefficient of friction is $1 / 1.0$ is the maximum and indicates that the 
thinning rate has its lowest value at this level of the parameter. Also, the relationships used by the software to calculate the SNR value for different modes are shown in Figure (30).

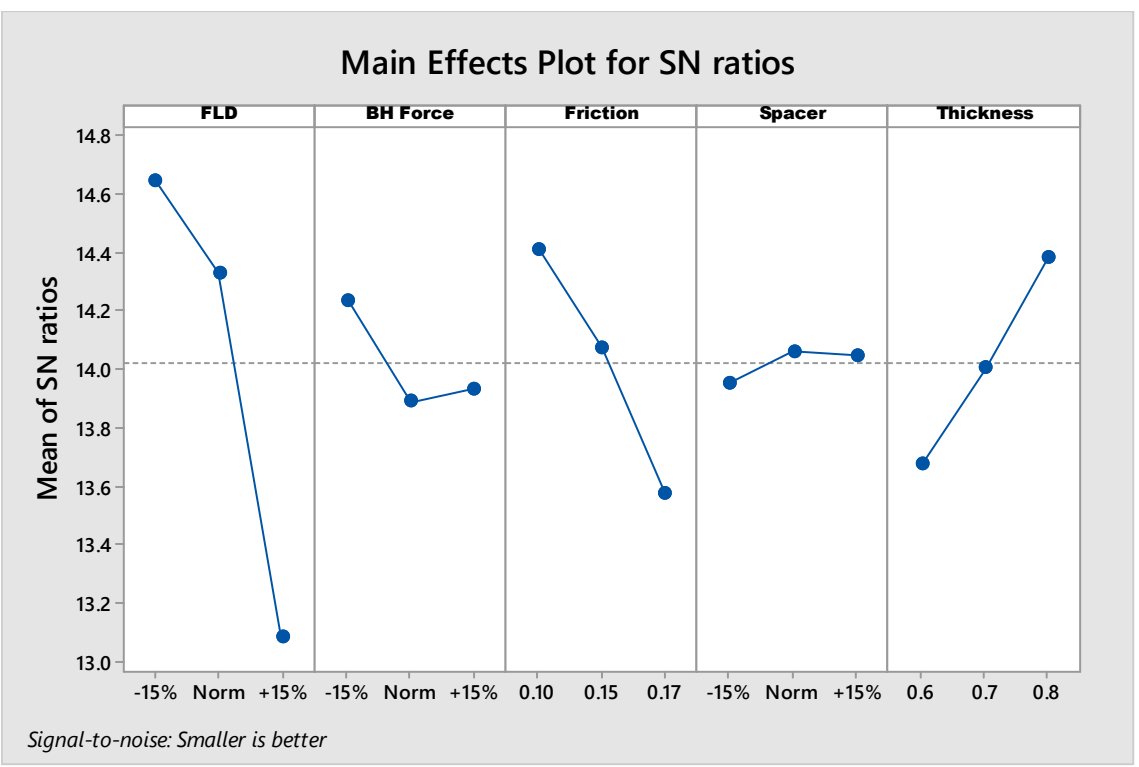

Fig. 29 Results of SNR analysis of Taguchi experiment
Signal to Noise Ratio:
Formula
$C$ Larger is better
$-10 \times \log 10\left(\operatorname{sum}\left(1 / Y^{\wedge} 2\right) / n\right)$
$C$ Nominal is best
$-10 \times \log 10\left(s^{\wedge} 2\right)$
C Nominal is best
$10 \times \log 10\left(\mathrm{Ybar}^{\wedge} 2 / \mathrm{s}^{\wedge} 2\right)$
(* Smaller is better
$-10 \times \log 10\left(\operatorname{sum}\left(\gamma^{\wedge} 2\right) / n\right)$

Fig. 30 SNR relations

\section{Critical zone No. 1}

As shown in Figure (31), with increasing initial sheet thickness, the thinning in this zone is significantly reduced.In the case of spacers or balancers, because their role is to control the flow of the sheet locally, in the case where their height is increased, and then the movement of the sheet is reduced, the sheet flow and consequently the thinning percentage decreases. As noted earlier, the role of friction is very significant compared to spacers or binder force in defects in sheet tearing. As the coefficient of friction increases, the thinness increases by about $2 \%$. This is because, in critical zone No.1, the sheet is not in contact with the die with the sheet holder and has a free formability. However, the areas around critical zone No.1 are in contact with the die and the sheet holder, and as the friction increases, the flow of the sheet in them becomes more difficult. As a result, the sheet in the critical zone is more stretched and thinner. With increasing binder force, the flow of sheet material decreases at the points where the sheet is located between the binder and the die. Therefore, a decrease in thickness change occurs at these points. But for areas that are freeflowing and under tension, such as walls that are being stretched, we expect an increase in the amount of thinning. Critical zone No.1 is also selected on a wall, which can be seen that with increasing binder force, the percentage of thinning in this zone has increased. But why the thinning 
has decreased slightly with a $15 \%$ increase in force is due to the predominant effect of the increase in thickness on the reduction in thinness. In other words, the increase in thickness has had a more significant impact than the increase in sheet strength.The forming limit diagram shows the critical combination of the two principal maximum and minimum strains, above which local throat instability is observed. This is well illustrated in Figure (31). With 15\% increase in the safe zone of the forming limit diagram, the thinning tolerance percentage increased significantly by $4 \%$. Examples are Experiments 1, 16, and 22 listed in Table (2), in which there are three levels of FLD examined (Experiment No.1, Low Limit, Experiment No.16, Normal, and Experiment No.22 The upper limit of the FLD chart is set) has been applied, noted. It is worth noting that these three experiments have been selected in such a way that the effect of other parameters is in line with the subject under study. In these experiments, while the percentage of thinning has increased, which has been associated with a decrease in the amount of rupture area. The illustrations for these three experiments are shown in Figure (32). As seen in the image, the thinning rate in experiments 1,16 and 22 was $18,18.7$, and $22.5 \%$, respectively. This could mean that by making changes to the properties of the sheet and achieving a higher FLD, one can expect a more desirable formation with less thinning of the sheet.

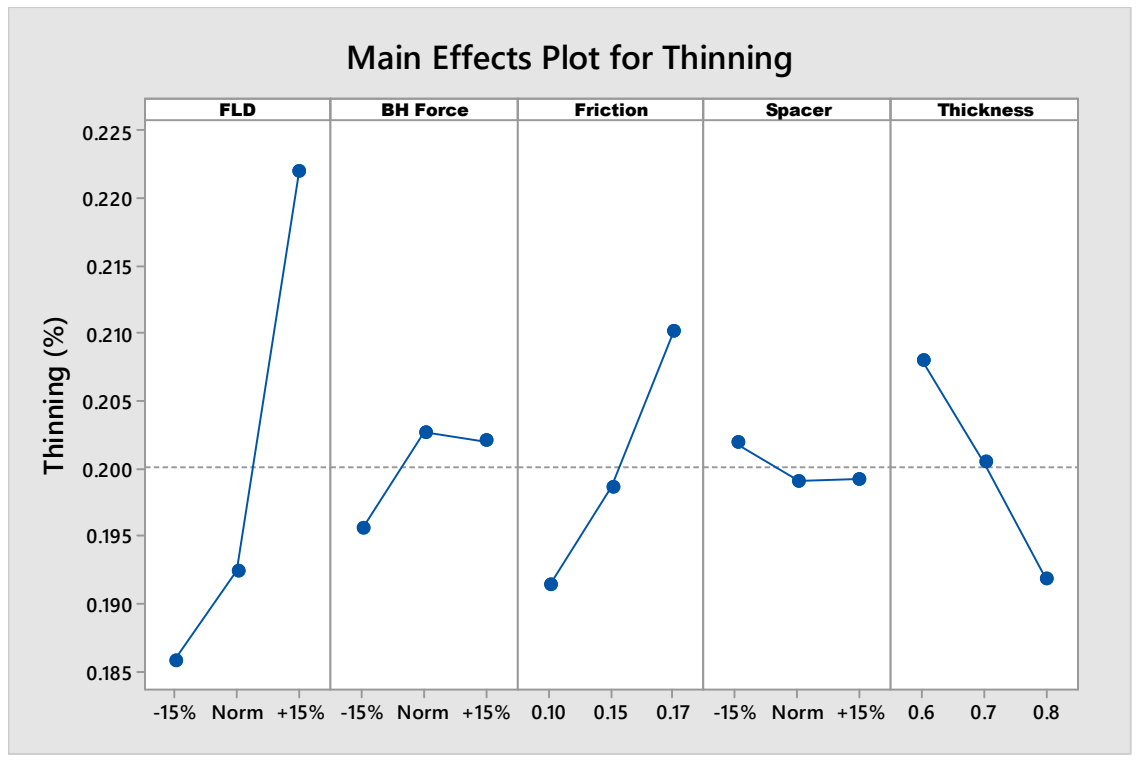

Fig. 31 Percentage of thinning of critical zone No. 1 


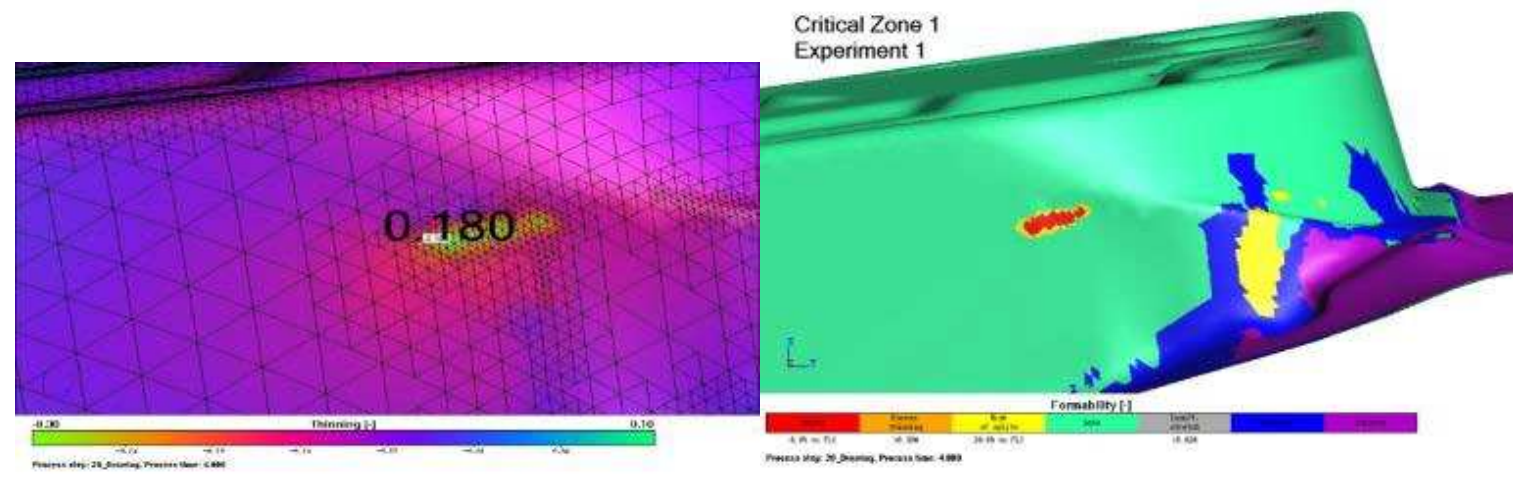

(a)

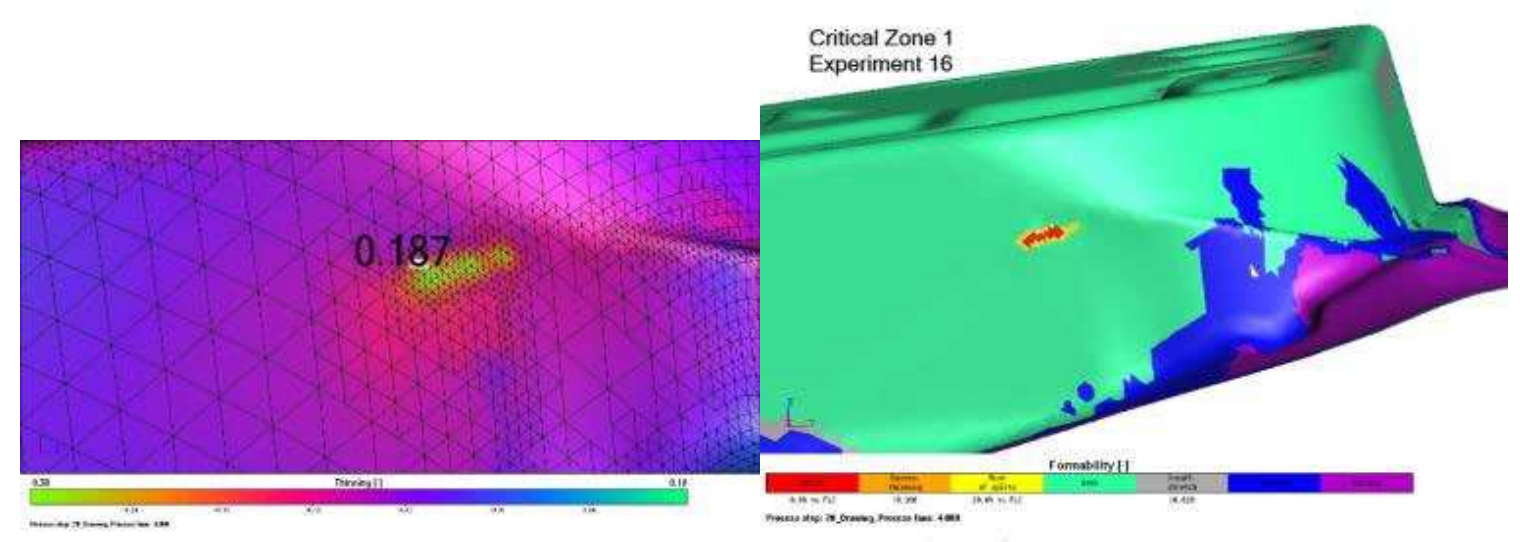

(b)

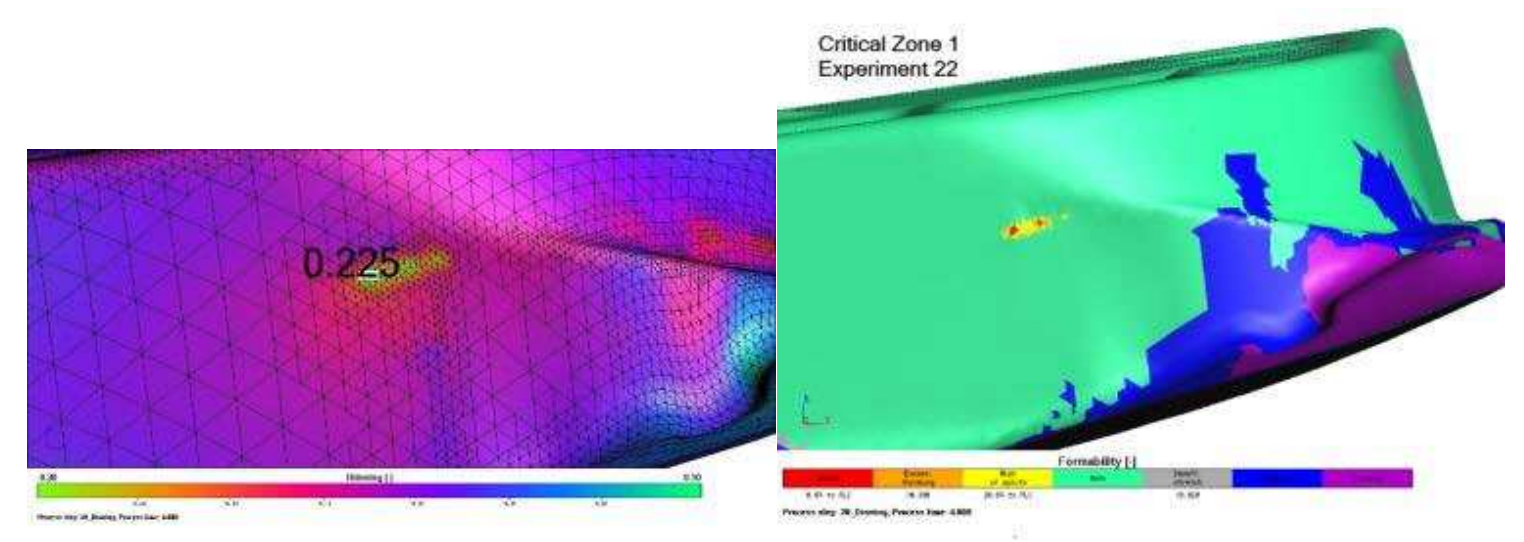

(c)

Fig. 32 (a) Experiment No.1 with 15\% reduction in ductility, (b) Experiment No.16, ductility of DC04 sheet, (c) Experiment No.22 with 15\% improvement in ductility and percentage of thinning in critical zone No.1 
The interaction of the coefficient of friction and the initial thickness of the sheet used can be seen in Figure (33). In general, with increasing thickness, the amount of thinning has decreased, but this decrease in thinning has decreased less with increasing the coefficient of friction, which shows that friction has a more tangible effect on the amount of thinning compared to the thickness of the sheet. On the other hand, the steep slope of the diagram in the two curve intervals related to the thickness of 0.6 , while the coefficient of friction has increased to a lesser extent $(0.02$ compared to 0.05 ), will indicate that at smaller sheet thicknesses, the effect of the coefficient friction is more.

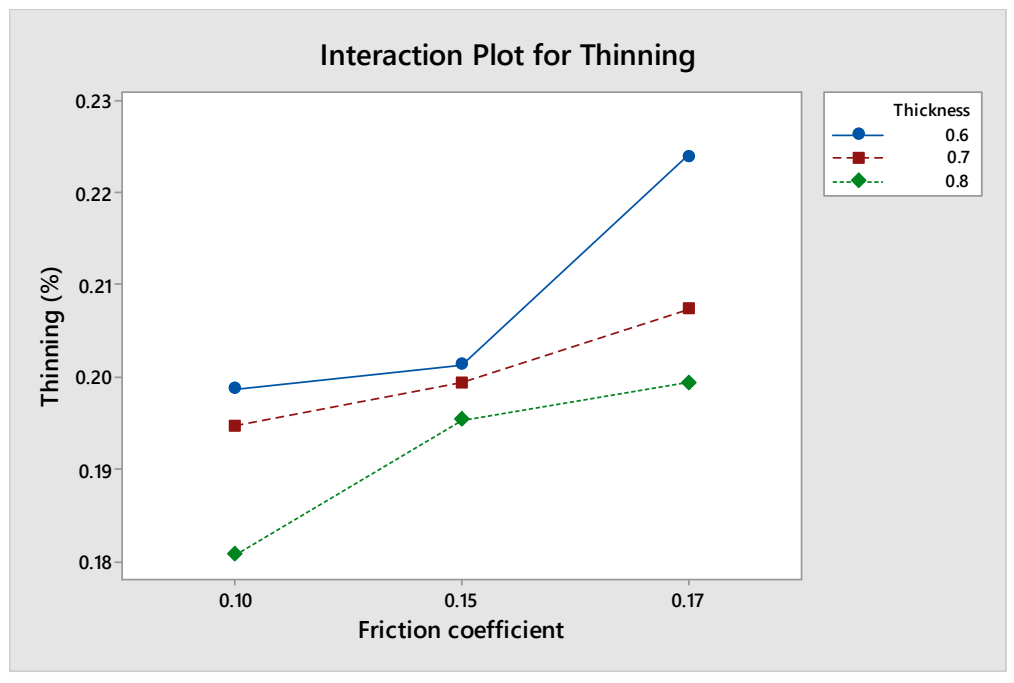

Fig. 33 Interaction of friction coefficient and thickness on the percentage of thinning of the critical zone 1

Fig. 34 shows the interaction of FLD and the coefficient of friction. As can be seen, with increasing coefficient of friction, the percentage of thinning has also increased. However, higher FLDs have also increased the thinning tolerance of the sheet. This means that by having a sheet with improved properties, the desired part can be reached with higher friction coefficients if necessary.

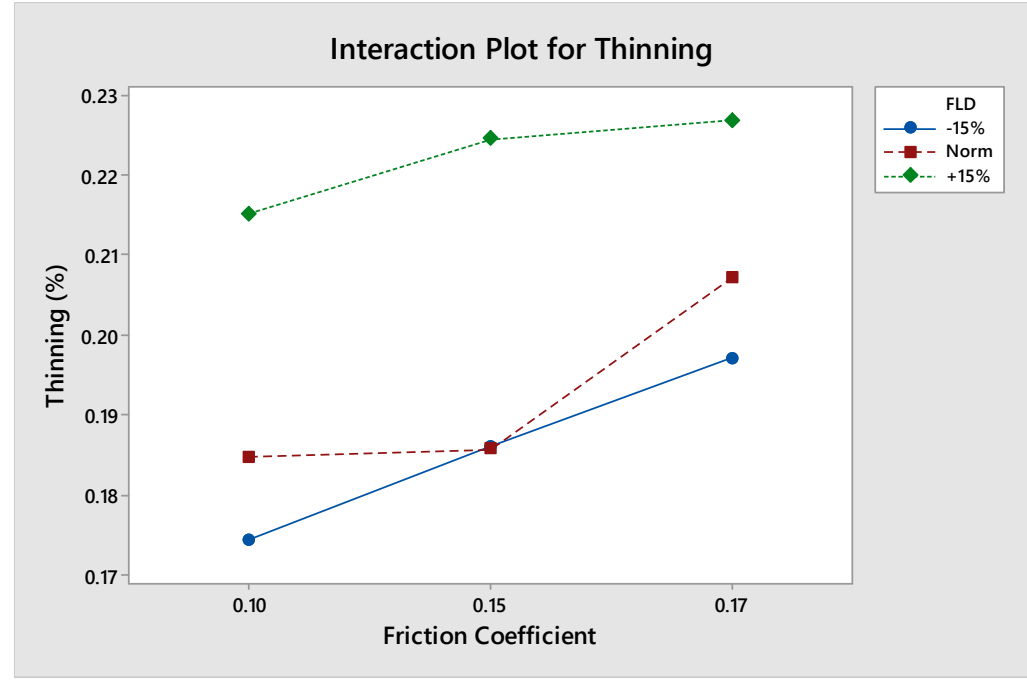


Fig. 34 Interaction of friction coefficient and FLD on the critical thinning percentage 1

The diagram of Figure (4-11) is shown the interaction of thickness and position of forming limit diagram. It can be seen that despite the decrease in thickness and increase in thinning, this FLD is higher, which has still increased the thinning tolerance of the sheet so that the tensile process can continue without causing rupture.

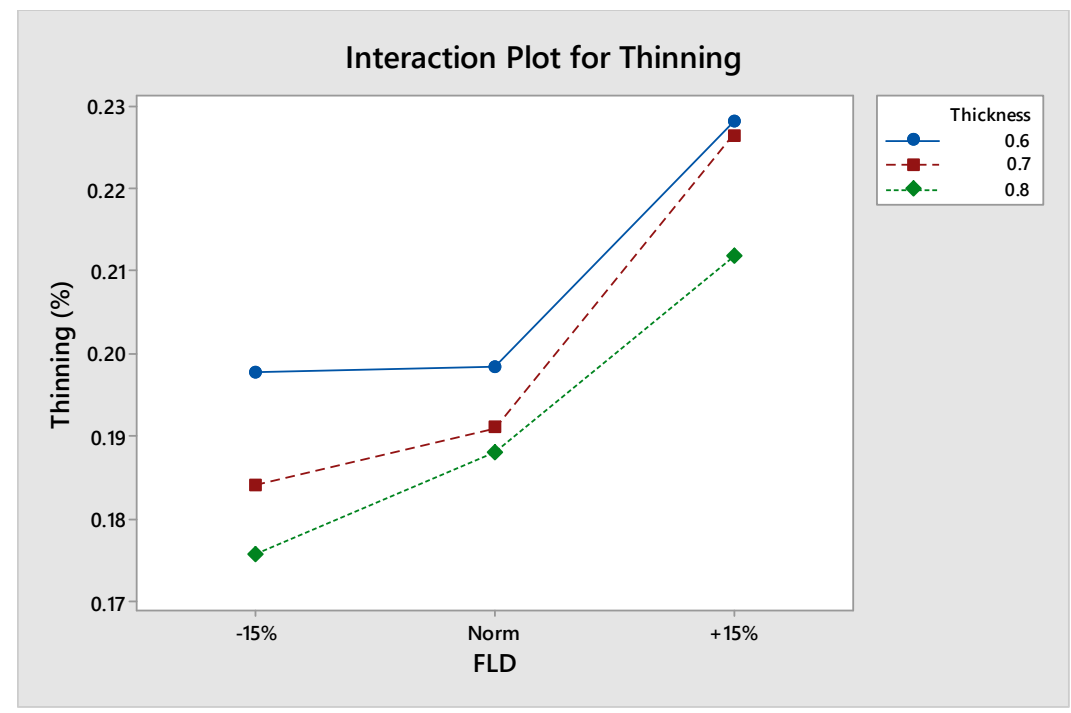

Fig. 35 Interaction of thickness and FLD on the critical thinning percentage 1

\section{Critical zone No. 2}

In this zone, the effect of thickness and friction coefficient parameters is similar to that stated in zone 1, but due to the position of this zone, which is farther from the edges of the sheet than region 1 , and also the lower tensile depth, resulting in the duration and the amount of contact of the sheet with the die has decreased, the thickness changes have shown a more significant impact against the binder force and FLD. In other words, due to the shallow depth of tension in this area, increasing the thickness has increased the resistance to thinning and improved it and has prevented the strain bearing capacity of the sheet from reaching. These effects can be seen in Figure (36). Regarding the lower amount of thinning at the lower height of the spacer, its interaction with the coefficient of friction should be considered. Figure (37). 


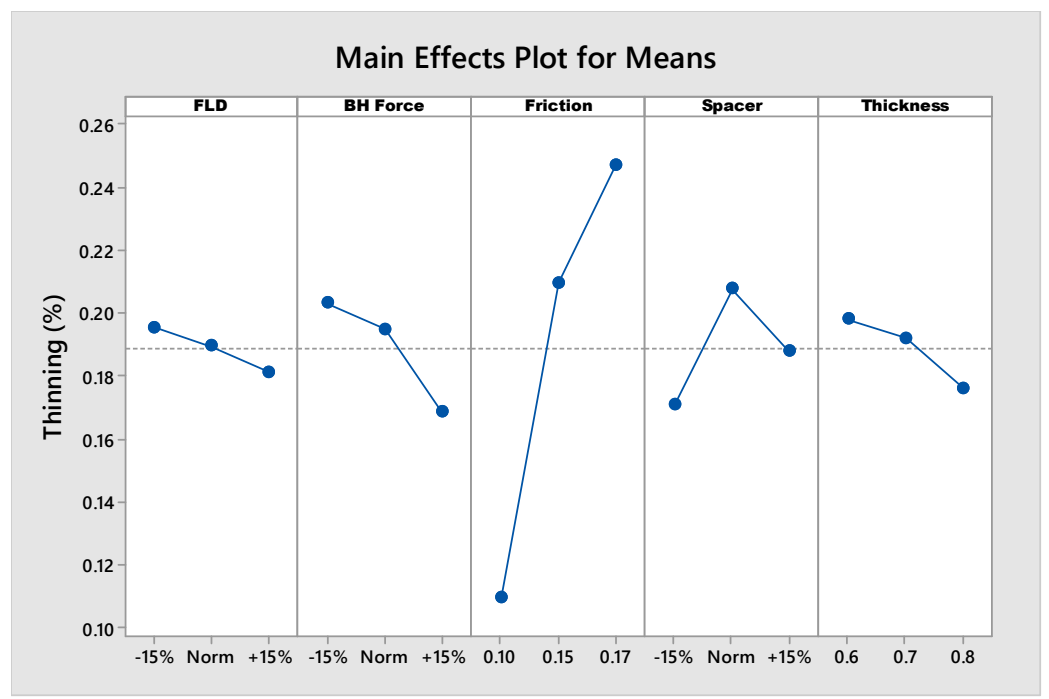

Fig. 36 Percentage of thinning of critical zone No.2

Regarding the interaction of spacers and friction, it can be seen that the significant effect of friction still prevails in standard and low spacer modes. So that at a coefficient of friction of 0.1 , changes in spacer height have not shown much effect on thinning, but with a $15 \%$ increase in spacer height, this effect has been adjusted by about 5\%. This means that increasing the height of the spacer has improved the impacts of friction-dependent thinning slightly.

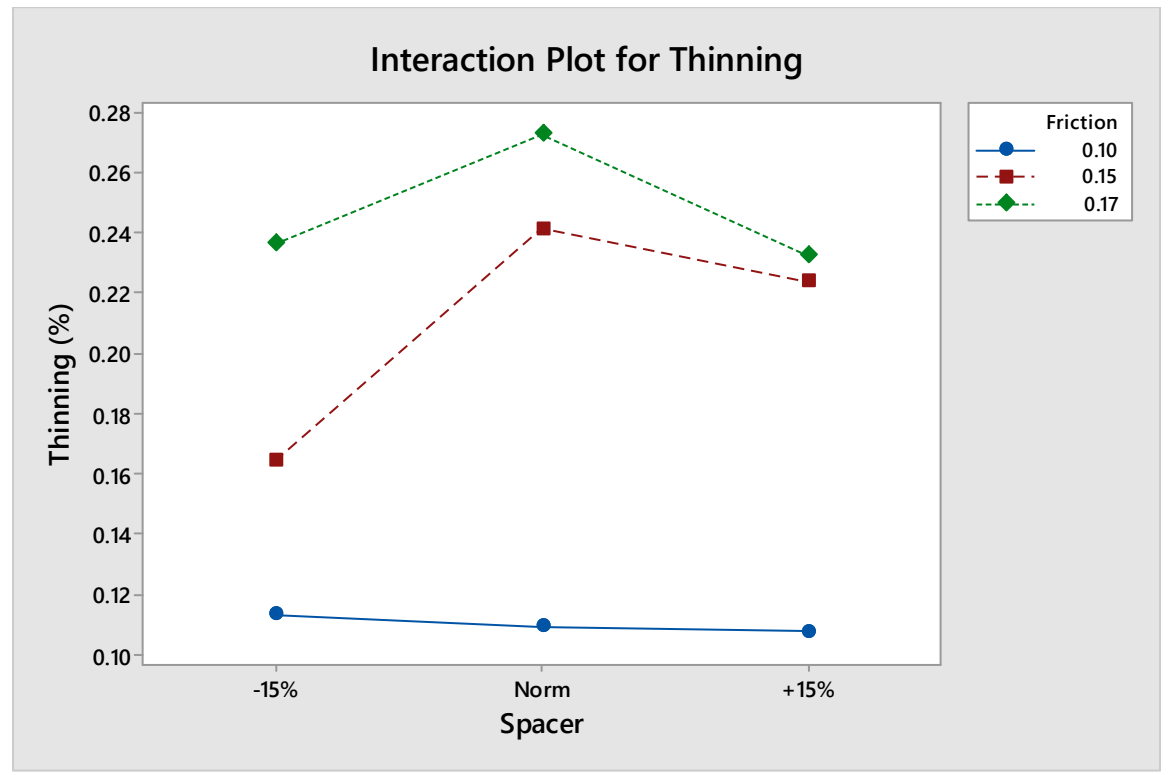

Fig. 37 Interaction of friction coefficient and spacer on the critical thinning percentage 2

\section{Critical Zone No. 3}

In zone 3, the results were similar to those presented in zone1, except that the intensity of the effect of the parameter changes was less. This is due to the non-uniform geometry of this zone compared to zone 1, so that a large percentage of sheet thinning occurs in walls with a high tensile depth around this area. On the other hand, according to Figure (38), which shows the geometry of the 
die in this area, the section marked on the image, after the formation of zone 3 , somehow shows the function of the binder and prevent more flow of the sheet around this area. These two factors have reduced the severity of thinning in Zone 3 relative to Zone 1, that shown in Figure (39).

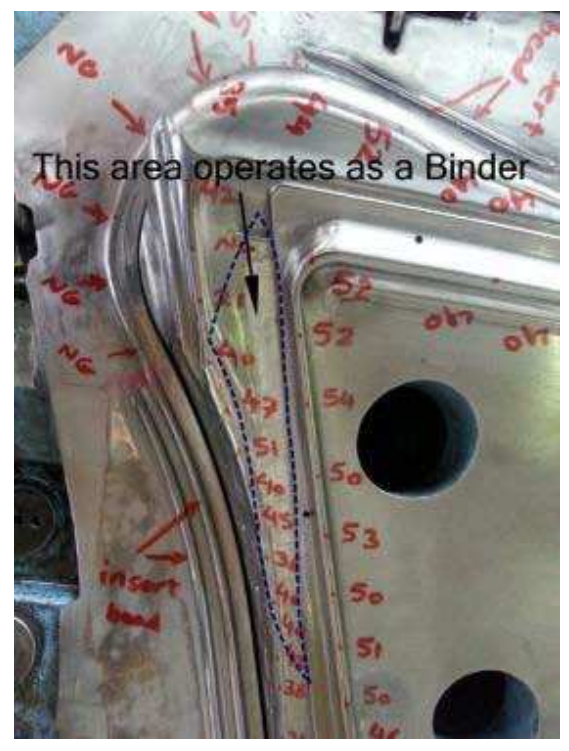

Fig. 38 Geometry around the critical zone 3

Also, with the explanations given about the geometry of this area, it should be said that the binder force has been affected in some way by the thickness changes. This is shown in Figure (40). As shown in the diagram, this is more effective at a thickness of $0.6 \mathrm{~mm}$.

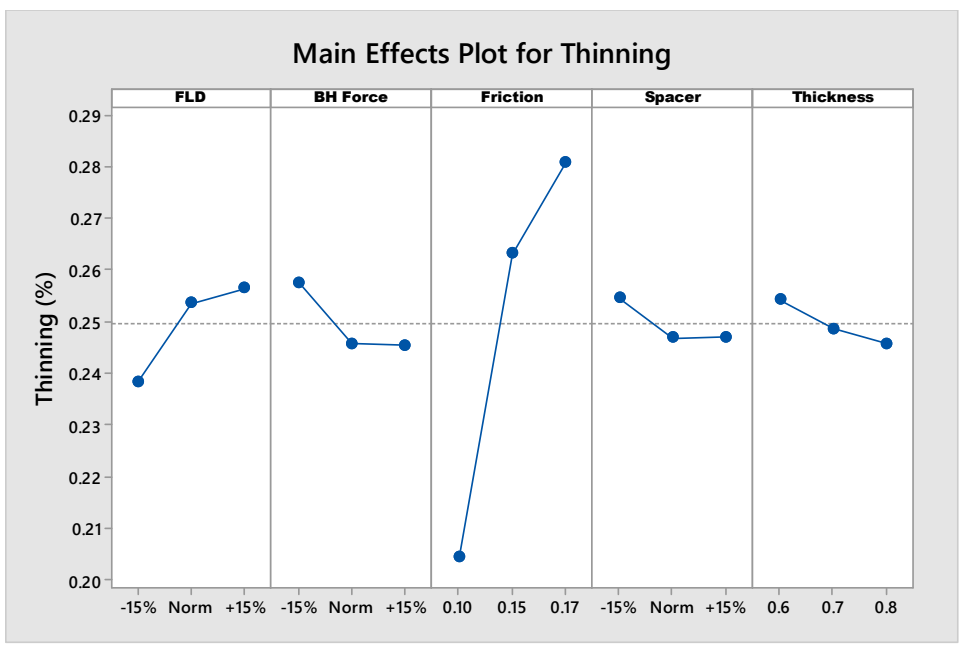

Fig. 39 Percentage of thinning of critical zone No. 3 


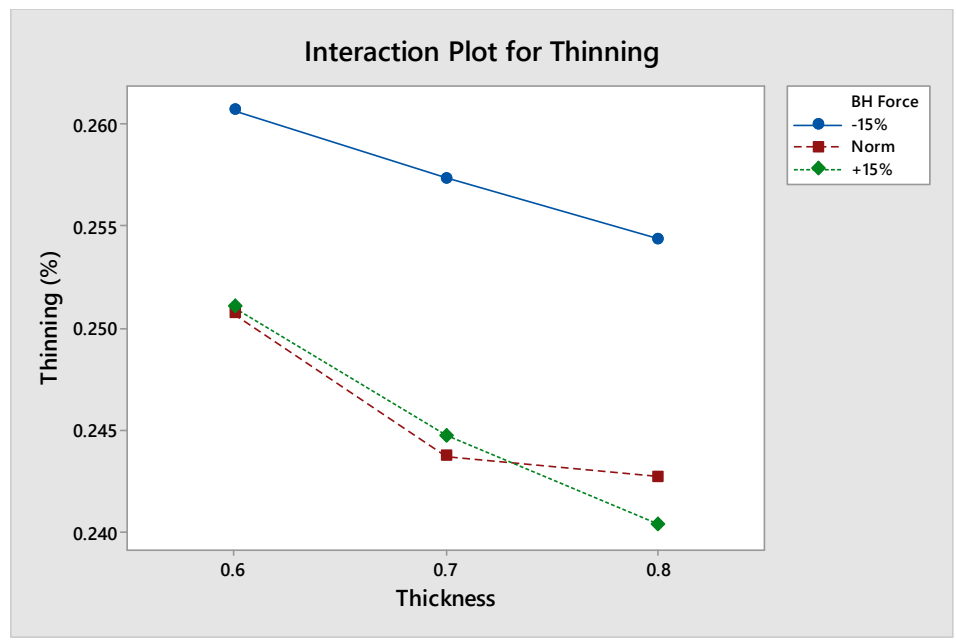

Fig. 40 Interaction of thickness and sheet strength on the critical thinning percentage 3

\section{Critical zone No. 4}

According to Figure (41), in the critical zone 4, the conditions were similar to critical zones 1 and 3 , with the difference that in the case of the binder force upper limit, according to what was discussed in zone 1, Areas A1 and A2 shown in Figure (42) become more elastic and therefore thinner. On the other hand, due to the position of this area, which is located on the filtered part, it is located between the two inlet paths of the material flow. In addition, zone A1 also acts as a binder and reduces most of the sheet flow cross-sectional, and then this flow increases in the wall with a high tensile depth and increases the thinning rate in the wall. It is noteworthy that this phenomenon did not occur in the lower limit of the binder force to its normal value because, in addition to increasing the thinning due to the increase of the binder force, other influential parameters also acted to increase the thinning rate. In other words, this thinning reduction due to the accumulation of material flow in critical zone 4 has been measured relatively (the intensity of the increase in thinning has decreased).

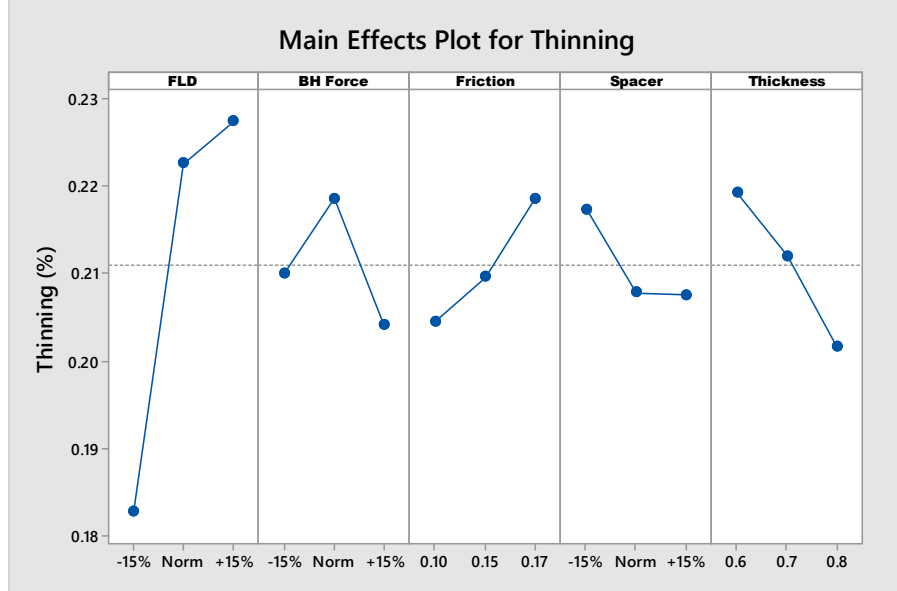

Fig. 41 Percentage of thinning of critical zone No. 


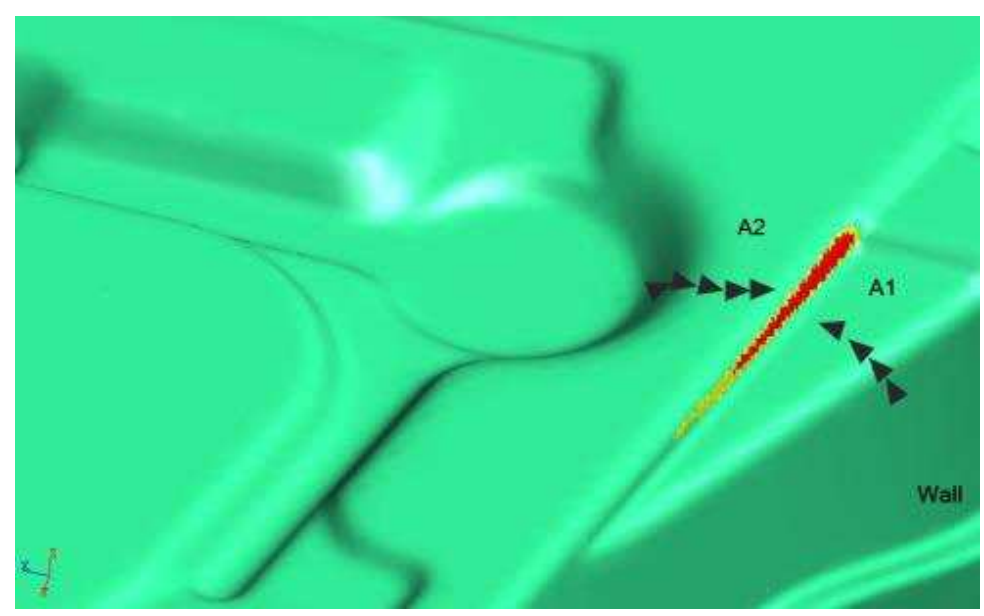

Fig. 42 Position of critical zone 4 and material flow path from surrounding geometry

\section{Investigation of the effect of parameters on surface strains}

This section investigates the behavior of the sum of the two primary minimum and maximum strains at the sheet surface. The reason for this is the zeroing of the sum of the surface strains and the thickness strain. Therefore, by decreasing or increasing the thinning rate, it is expected that the sum of the other two strains will decrease or increase so that there is always an equal relationship.

\section{Critical zone No. 1}

According to the diagrams (43) in this area, the part has less deformation with increasing thickness. This decrease also occurred with the increase of the height of the spacers due to the reduction of the sliding motion course. On the other hand, with increasing the coefficient of friction and the safe range of FLD, the deformation or the same strains on the sheet's surface has taken an upward trend. This is what showed the thinness of this zone in the form of a thick strain. But as can be seen, the changes in the shear force have not been able to have much effect on the surface strains. This is rooted in two issues. First, according to the diagram of the interaction between thickness and sheet strength in Figure (44), as it is clear, the changes in sheet strength versus thickness increase could not have much effect on the deformation of the part but was more affected. Increasing the thickness, which leads to a reduction in thinning, causes less deformation at the surface of the sheet. Secondly, according to what was observed in the thinning of this area, it can be seen that in this zone due to the increase of binder force, expansion occurred, and tension has a lower share in this area, so the deformation in the surface or the same Surface strains are reduced. 


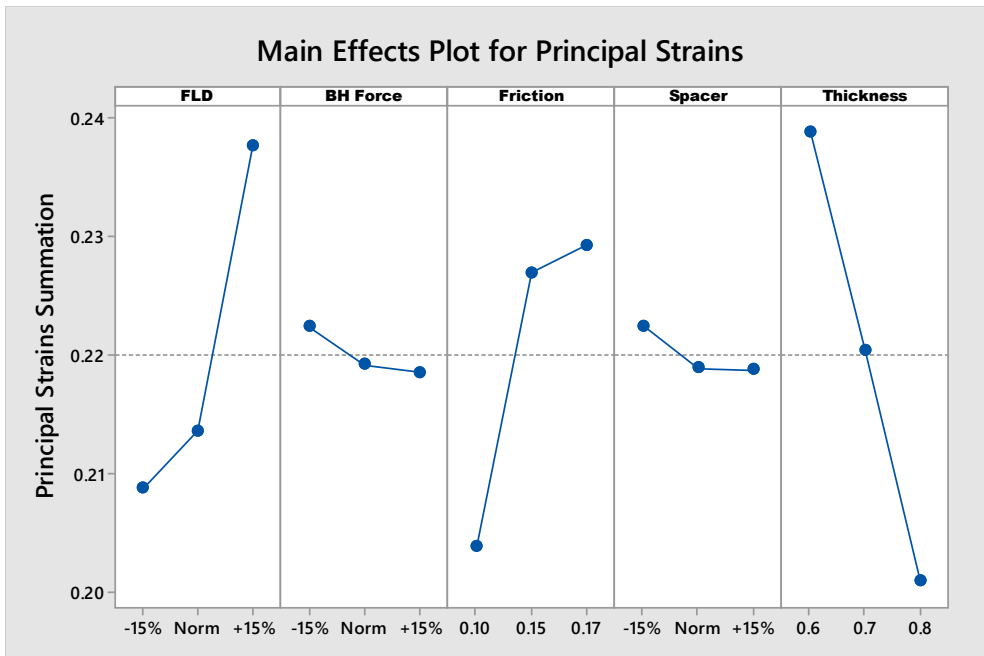

Fig. 43 The amount of main strains in the critical zone 1

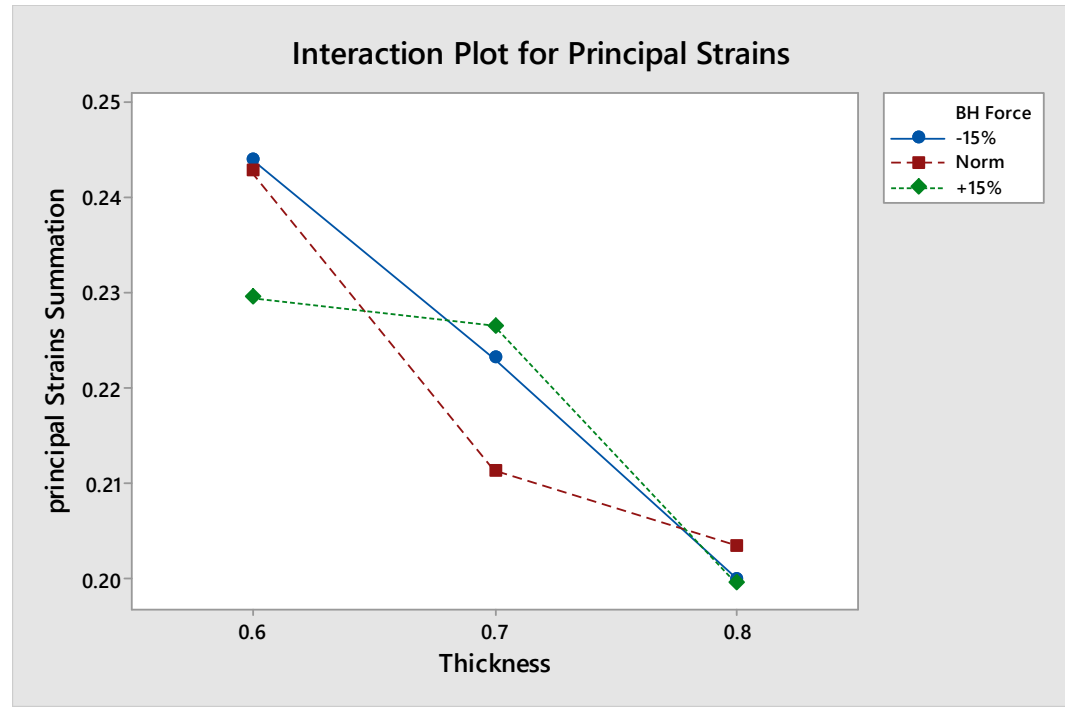

Fig. 44 Interaction of thickness and sheet force on the amount of surface strain in the critical zone 1

According to the diagram in Figure (45), it can be seen that with increasing FLD as well as coefficient of friction, the amount of surface strains has increased. As mentioned earlier, a higher FLD means that it can withstand higher strains before rupture or strangulation. In this case, too, it can be seen that the part has undergone more deformation without fracture. In addition, by comparing the slope of the diagrams, we find that in the case where a higher FLD range is used, at a similar level of variation of the coefficient of friction, the strain tolerance range of the material is further improved. 


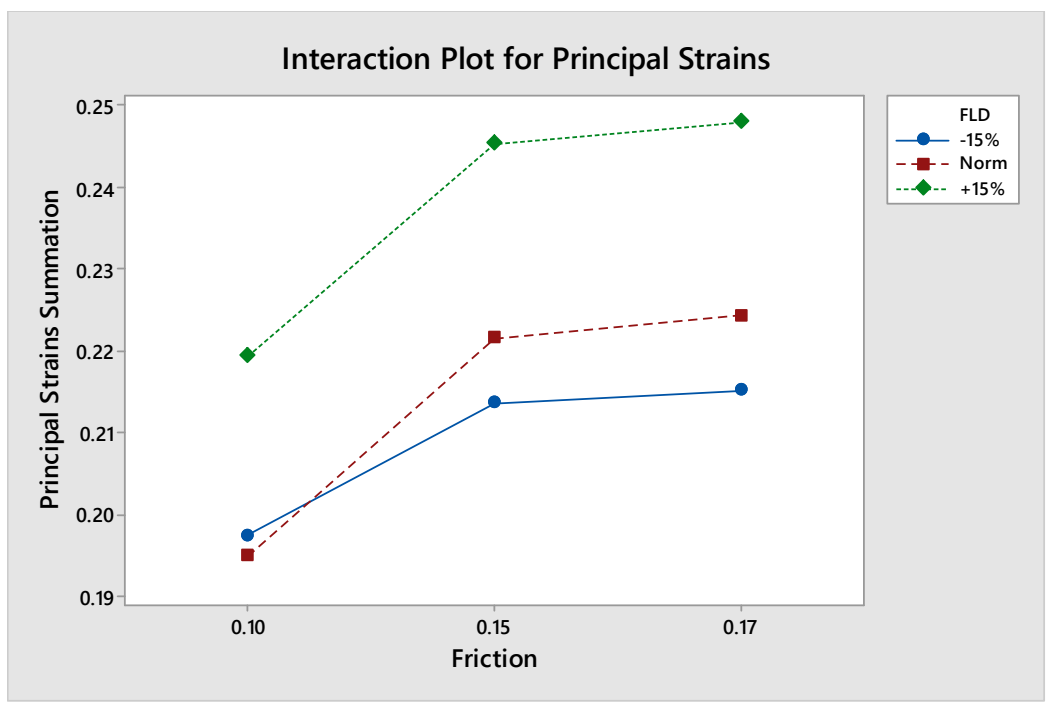

Fig. 45 Interaction of Friction coefficient and FLD on the surface strain of the critical zone 1

These changes can also be seen on the forming limit diagram in Figures (46) to (48). Light blue represents the elements of geometry, dark blue represents the values of the primary minimum and maximum strains in these elements, and yellow or red indicates the deformation path of the elements in the critical zone under study. Figure (46) shows the strain changes in region 1. As can be seen from the diagrams, the amount of strain decreases with increasing sheet thickness.

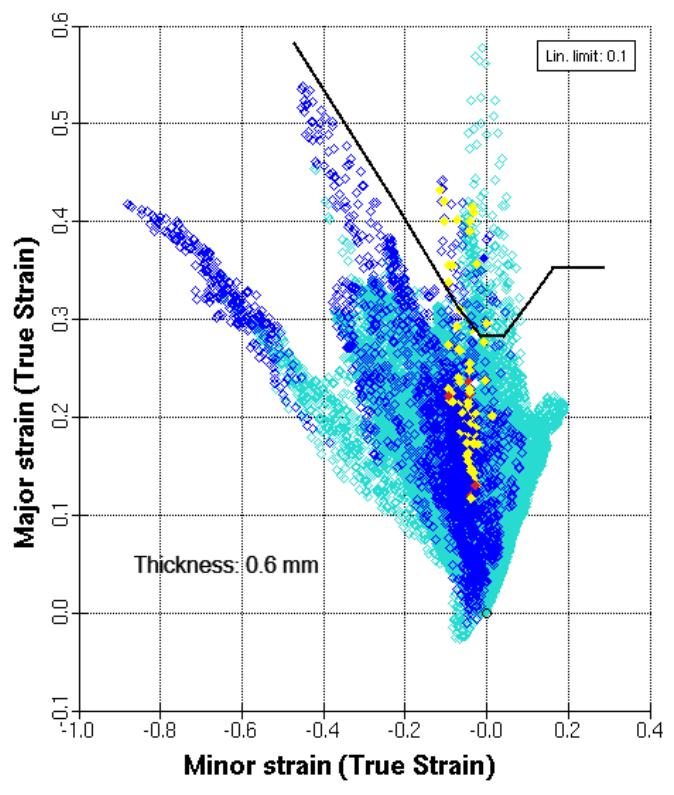

a

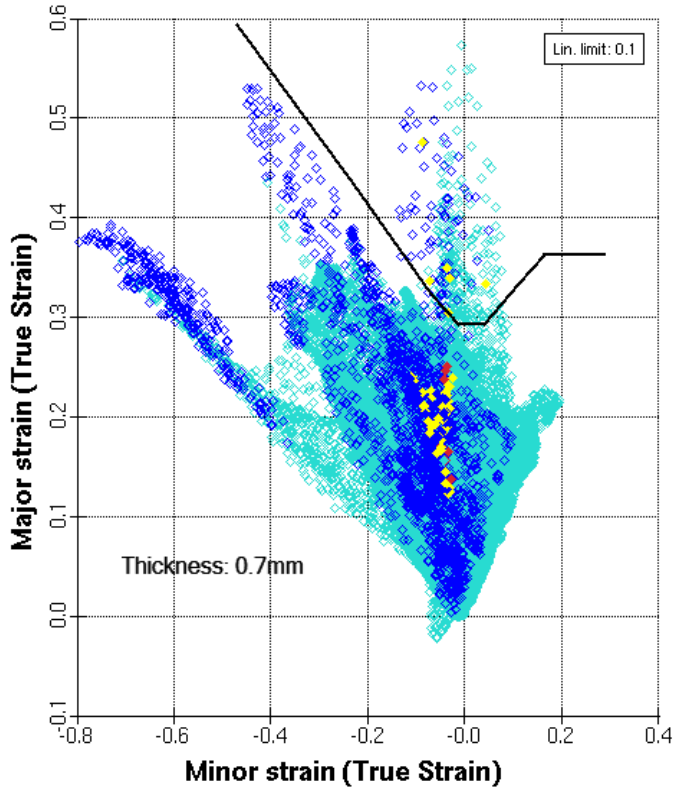

$\mathrm{b}$ 


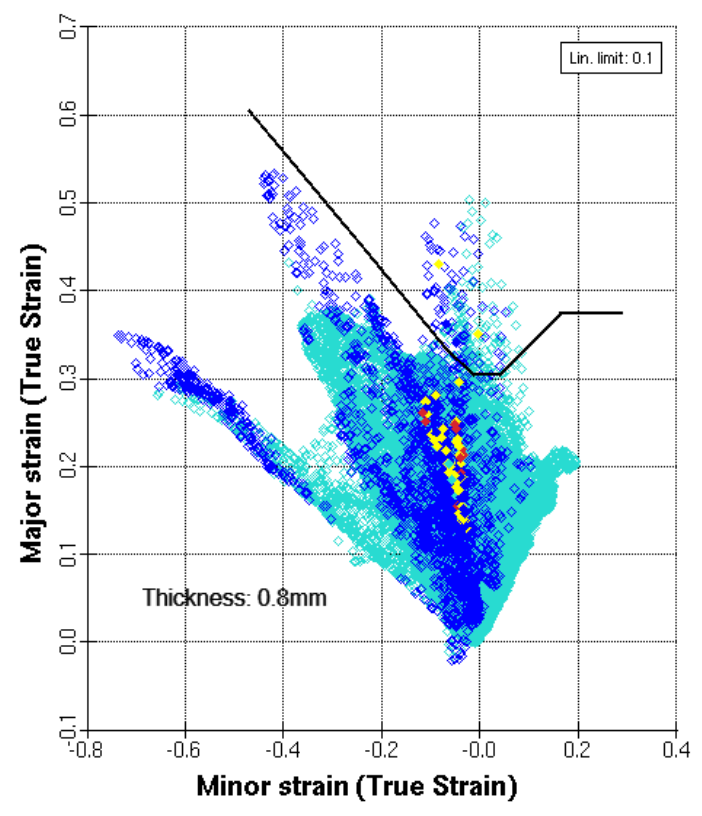

c

Fig. 46 Strain changes in critical zone No.1 in exchange for sheet thickness change as:

$$
\text { (a) } 0.6 \mathrm{~mm} \text { (b) } 0.7 \mathrm{~mm} \text { (c) } 0.8 \mathrm{~mm}
$$

Also, the strain changes in this zone in exchange for the FLD changes and the height of the spacers are shown in Figure (47). As can be seen, with increasing FLD range, the sheet's strain tolerance and its deformation have increased, and the sheet has failed at higher strains.

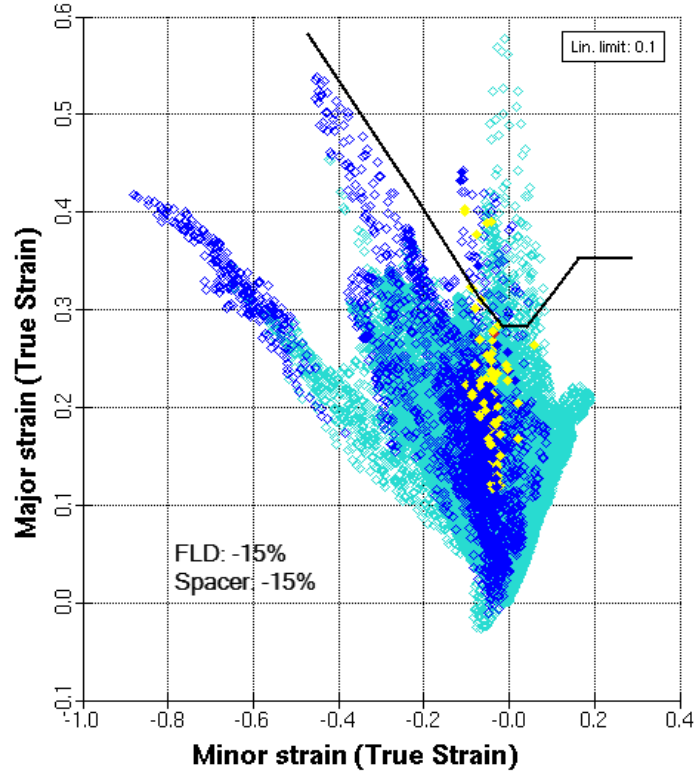

a

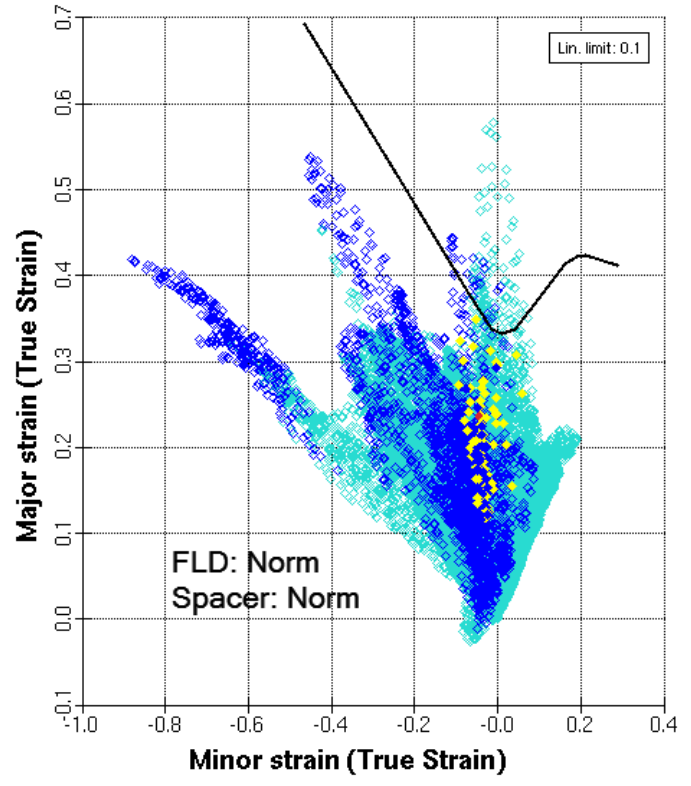

$\mathrm{b}$ 


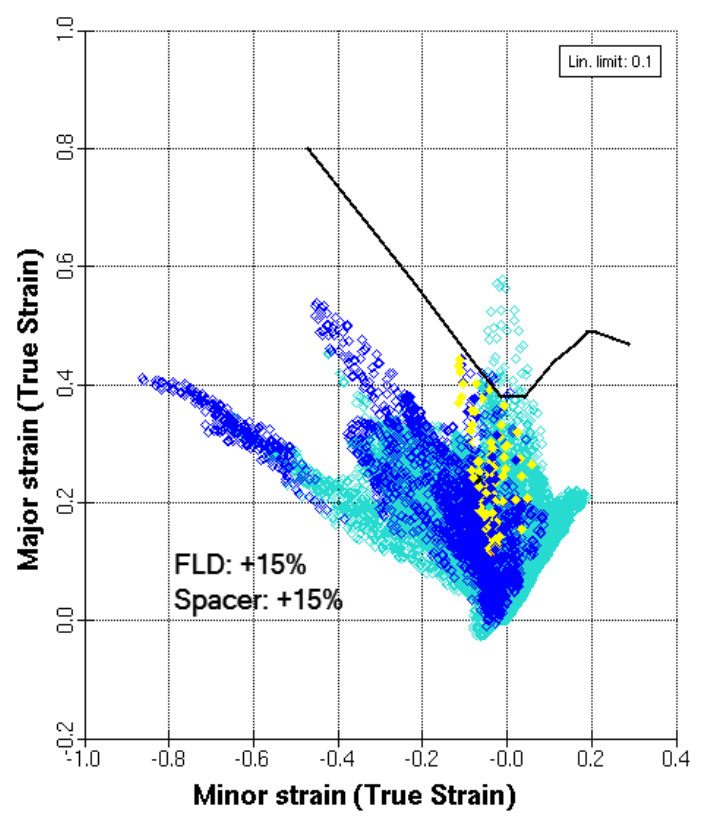

C

Fig. 47 Strain changes in critical zone No. 1 for: (a) 15\% decrease in ductility and height of spacer, 2 (b) normal state, and (c) 15\% increase in ductility and height of the spacer

The effect of friction on changes in strain rates is also shown in Figure (48). It is observed that with increasing the coefficient of friction, the amount of strains has increased significantly. In addition, the distribution of strains in this region has become more uniform when the coefficient of friction is 0.1 .

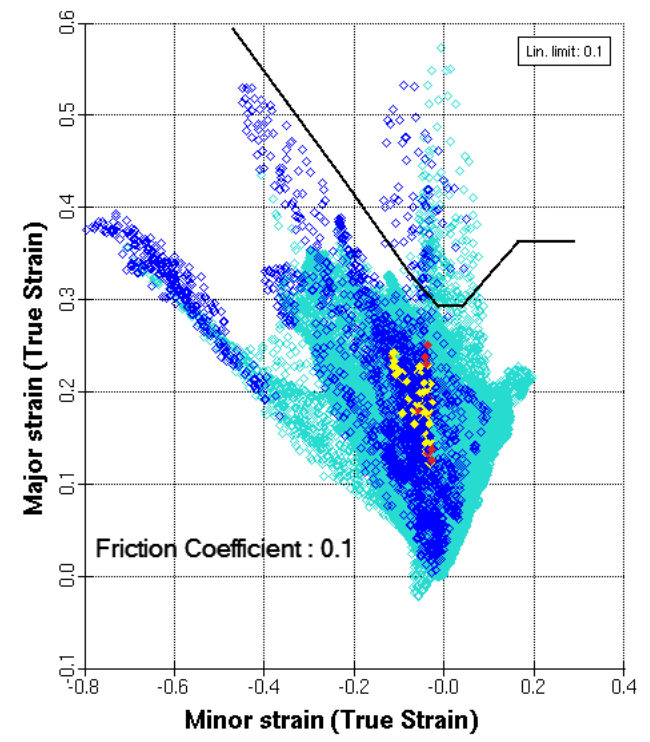

a

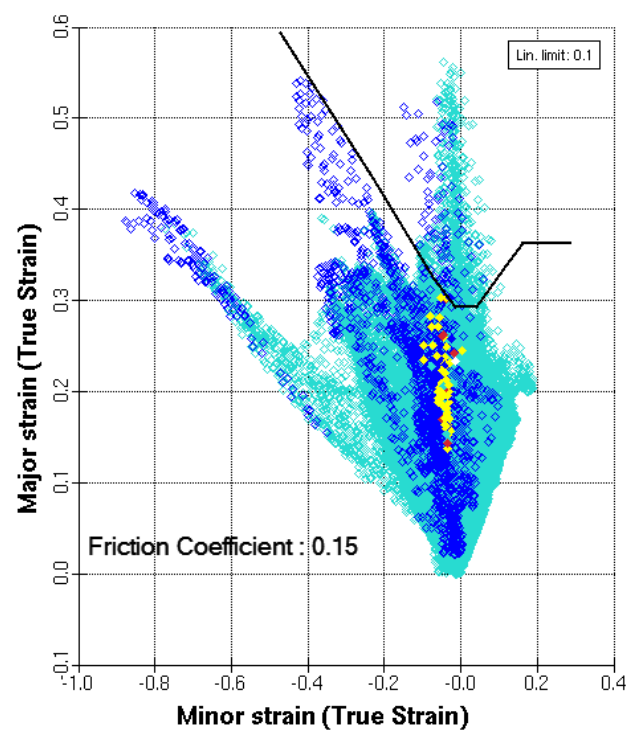

b 


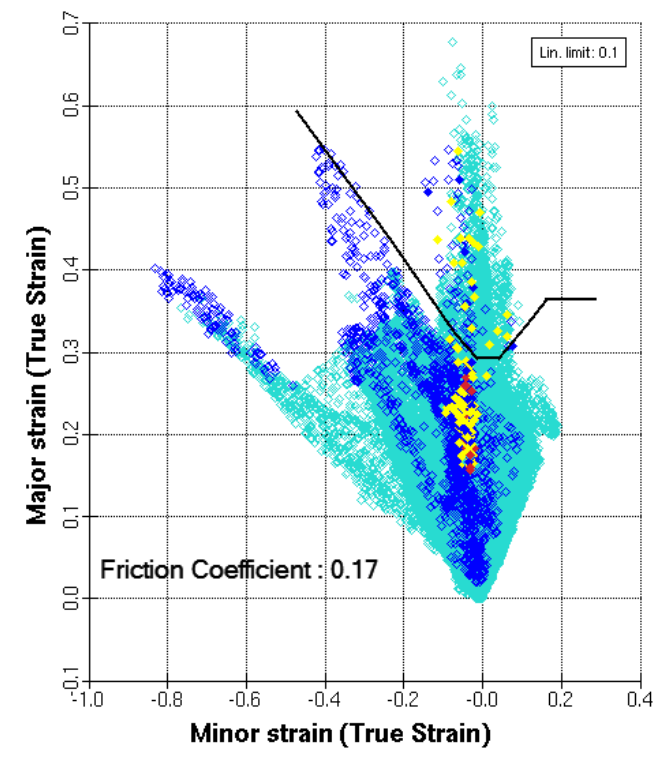

c

Fig. 48 Strain changes in the critical zone No. 1 in exchange for the coefficient of friction change (a) 0.1 , (b) 0.15 , and (c) 0.17

\section{Critical zone No. 2}

In this region, the trend of thickness, friction, and sheet force was similar to that of zone 1. Regarding the changes in the amount of strains in exchange for changes in FLD, it should be said that due to the shallow depth of tension in this zone and its position, the deformation rate is not so high that the higher FLD can show its effect. It is also affected by the impact of other parameters in the experiment, such as increased thickness as shown in figure (49).

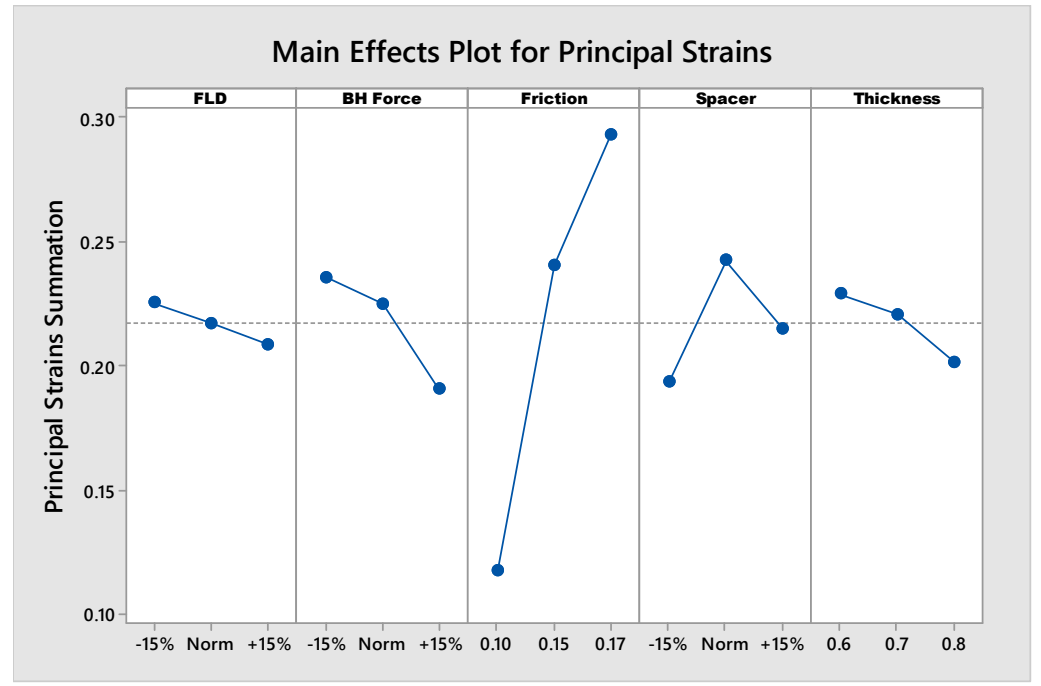

Fig. 49 The amount of main strains in the critical zone 2 
In Figure (50), it can be seen that the amount of surface strain increases by increasing the coefficient of friction. An increase in the height of the spacers is adjusted to a higher level. In other words, by increasing the height of the spacers by $15 \%$, the sliding motion course decreased, and consequently, the effect of friction on the deformation rate decreased.

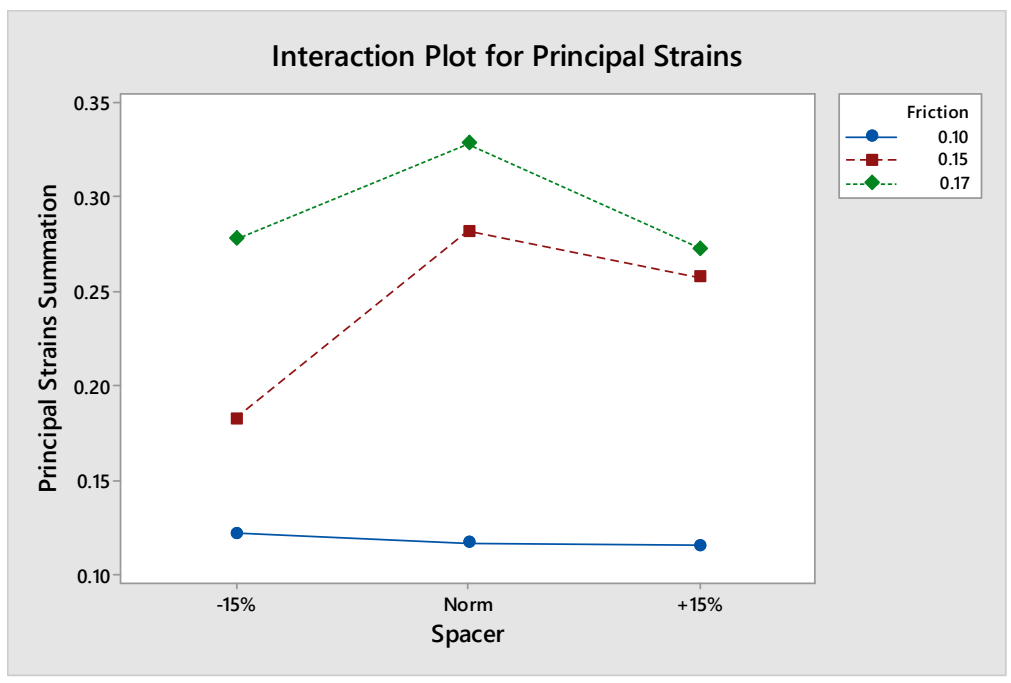

Fig. 50 Interaction of friction coefficient and spacer on the amount of surface strain in the critical zone 2

\section{Critical zone No. 3}

According to the diagrams in Figure (51), the trend of strain changes in this region was similar to that of region 1, but due to the different geometry in this region, unlike region 1, which is drawing as a wall, and also according to what is in section 4. 2-3 Regarding the geometry around this area, it has been said that the stresses applied to the sheet are distributed in different directions, or their amount is reduced, and the intensity of the strain changes is reduced.

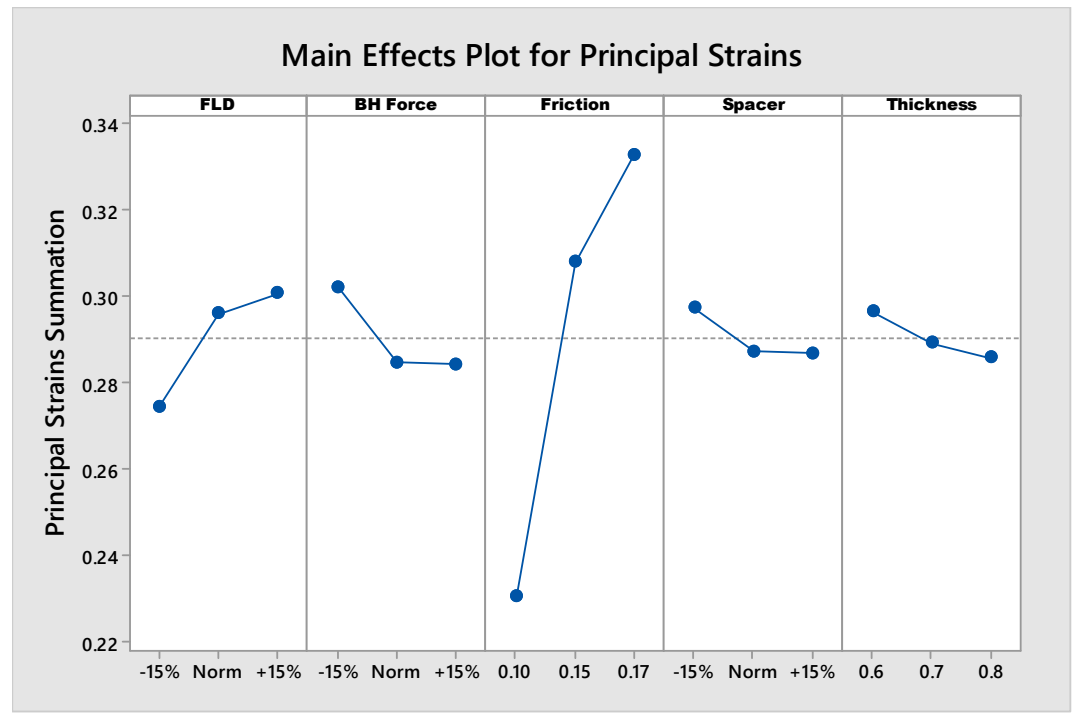

Fig. 51 The amount of main strains in the critical zone 3 


\section{Critical zone No. 4}

In this zone, the trend of strain changes was similar to that in zones 1 and 3 . However, as mentioned about the effect of binder force changes on the thinning of the walls, with increasing binder force, the amount of deformation in the wall shown in Figure (52) increased and despite the A1 zone between the wall and the critical zone 4, Reduces the continuation of the increasing trend of strain in this area. In addition, due to the position of this zone, which is located on the filleted section between two horizontal surfaces, it can be said that there is practically no significant deformation in the surface of this zone. Figure (53).

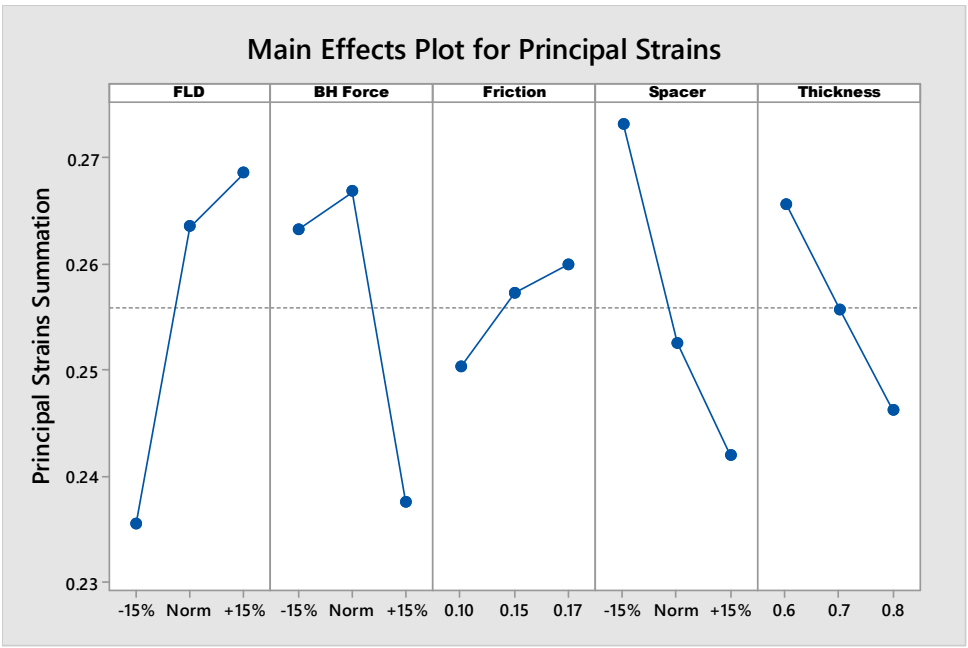

Fig. 52 The amount of main strains in the critical zone 4

According to Figure (53), it can be seen that increasing the strains due to higher FLD and consequently increasing the strain tolerance of the sheet has a greater effect than reducing the deformations that occur due to increased thickness.

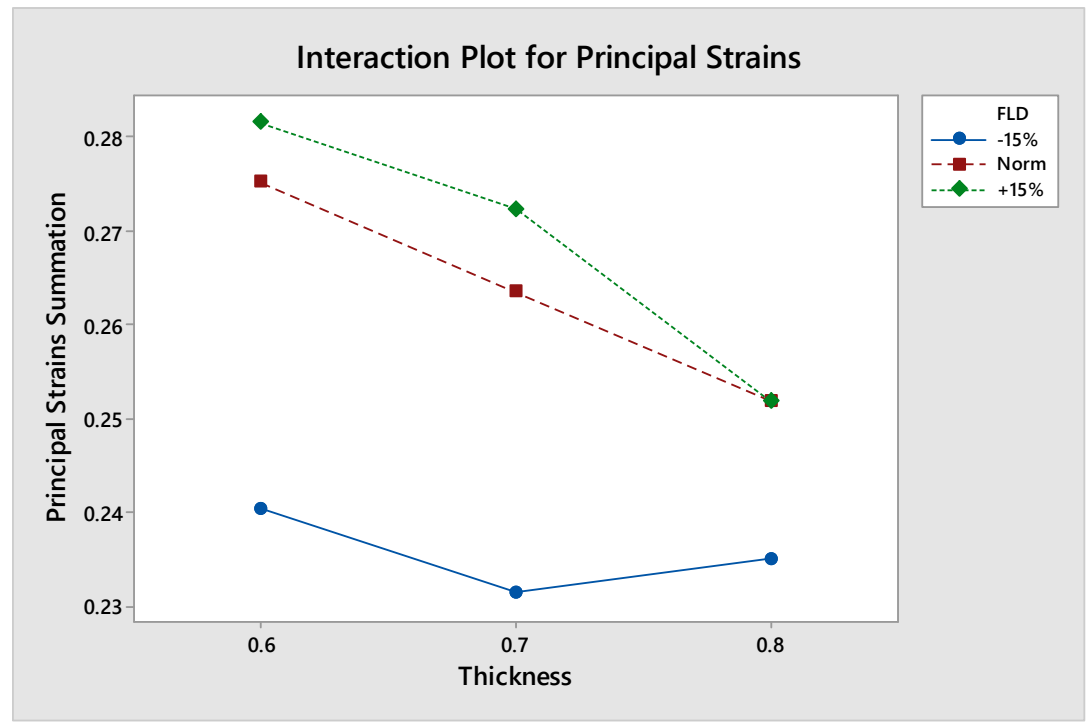

Fig. 53 Interaction of thickness and FLD on the surface strain of the critical zone 4 


\section{Investigating the trend of strain changes in the final part}

To validate the results obtained from the experiments, the distribution of thickness and surface strains in exchange for changes in some of the parameters at the end of the drawing process is given. It seems necessary to mention that in the images related to surface strains, the length and color of the vectors indicate the intensity or rate of strains in that area, which is shown in Figure (54).

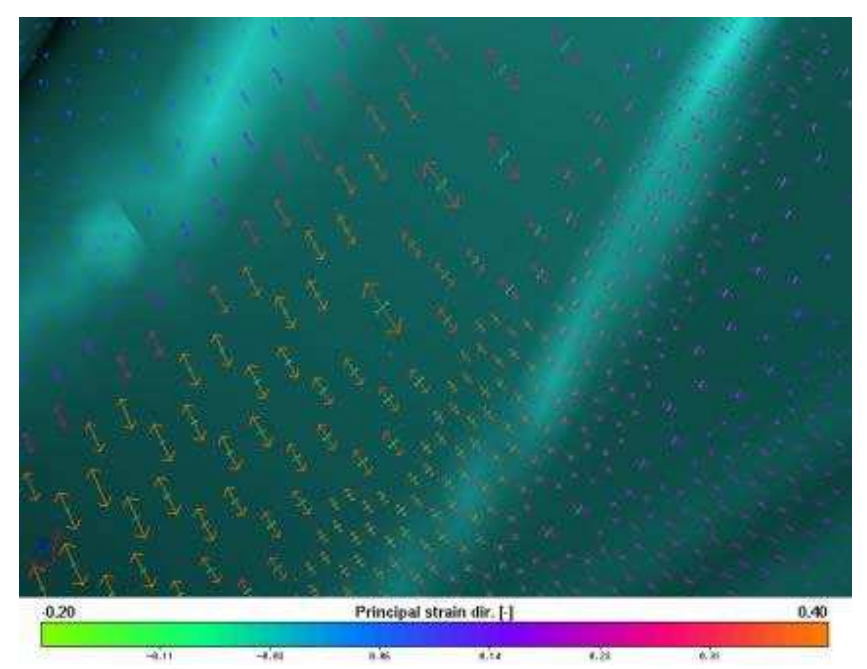

Fig. 54 Vectors represent the distribution of surface strains

Figure (55) shows how the thickness is distributed in exchange for changes in the coefficient of friction. As can be seen, with increasing the coefficient of friction, the amount of thinning has increased, and even in the critical zone that the software had predicted, the part has been torn.

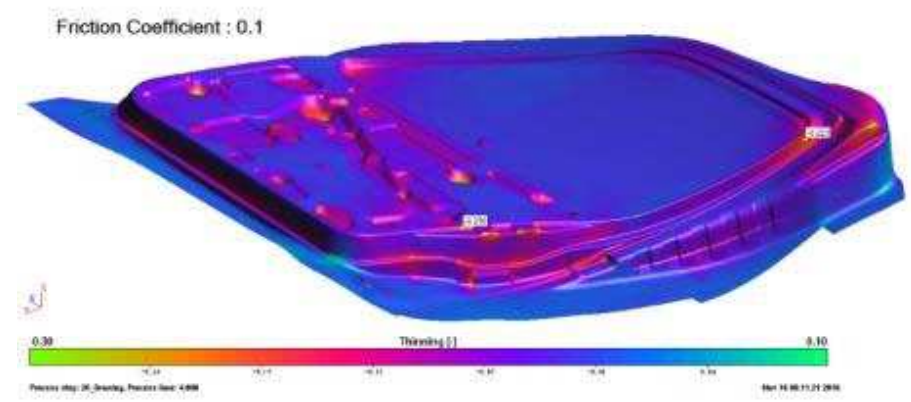

(a) 


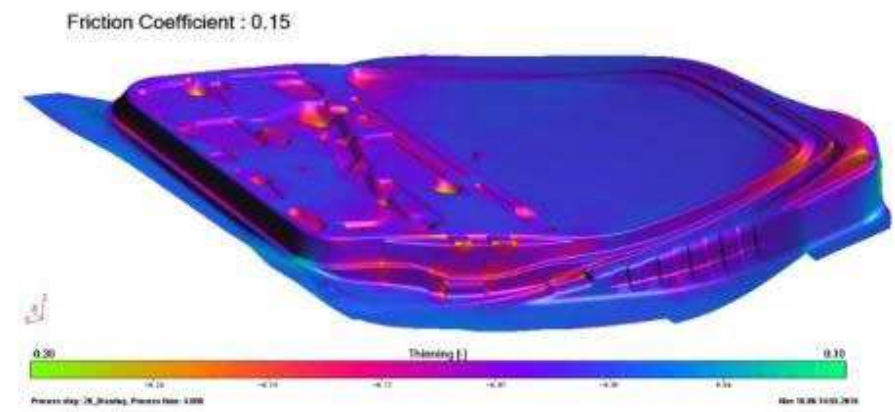

(b)

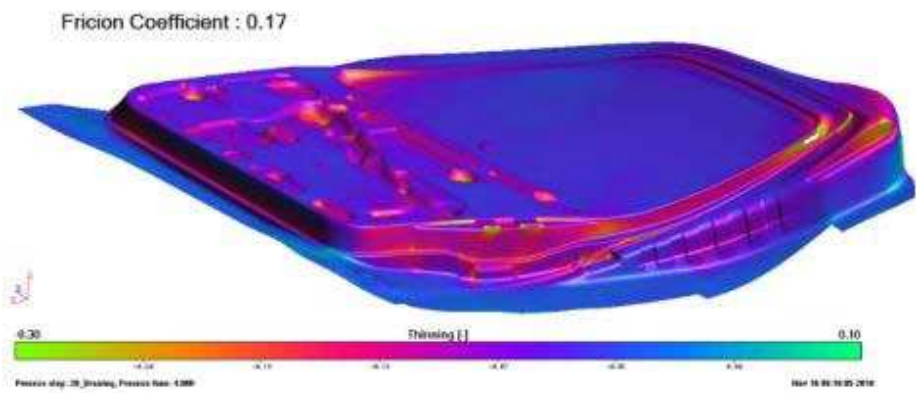

(c)

Fig. 55 Distribution the thickness in the values of (a) 0.1 , (b) 0.15 , and (c) 0.17 of the coefficient of friction

Also, in Figure (56), the coefficient of friction shows the intensity and direction of the principal strains at the tested surfaces. As can be seen, the strain rate is higher in the high-risk areas predicted by the software and the areas where the material flow has accumulated. According to the images, the amount of these strains has also increased by increasing the friction coefficient.

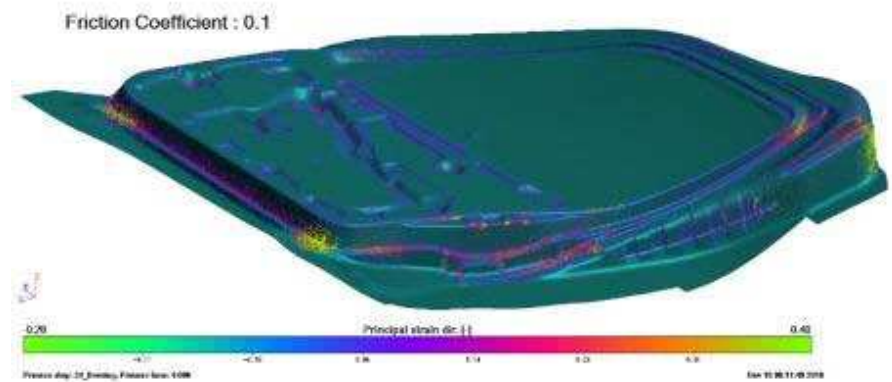

(a) 


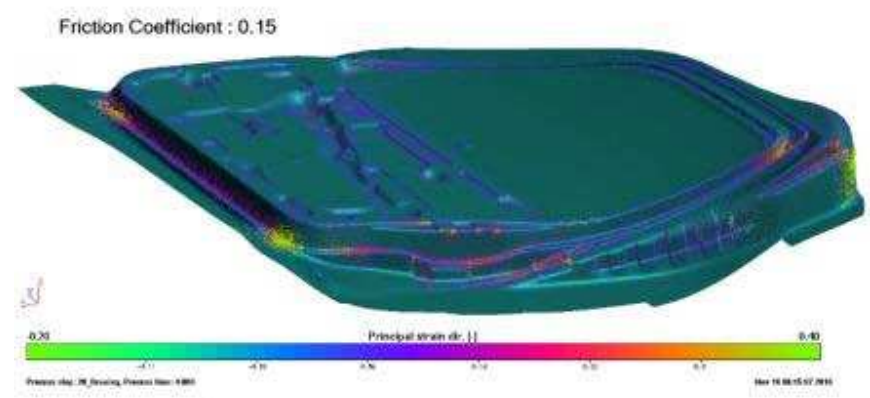

(b)

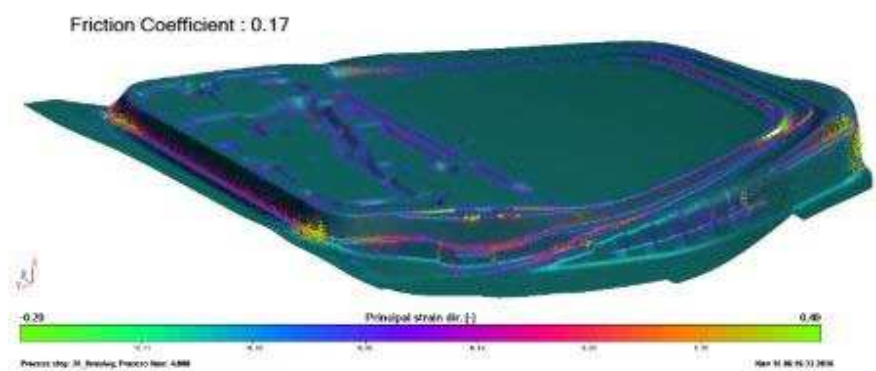

(c)

Fig. 56 Distribution of the principal strains in the values of (a) 0.1 , (b) 0.15 , and (c) 17.0 of the coefficient of friction

In Figures (57) and (58), it can be seen that the increase in the strain tolerance of the sheet before rupture occurred as a result of increasing the safe range of the forming limit diagram. Figure (433) shows the thickness strain distribution at different levels of the FLD diagram and spacers. Another issue deduced from this figure is the more significant effect of FLD changes on strains than changes in spacer height.

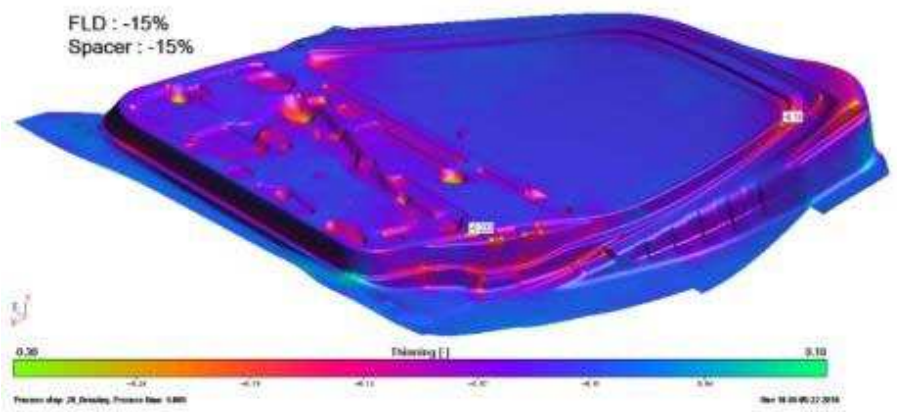

(a) 


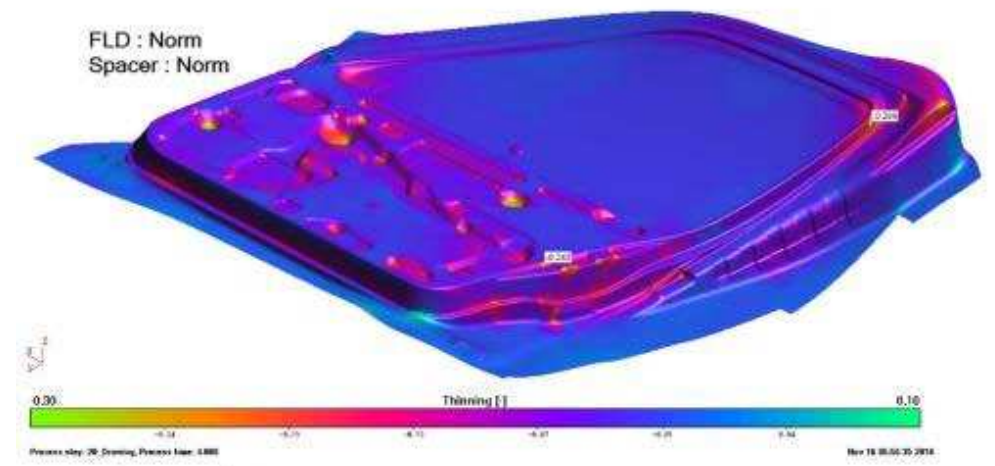

(b)

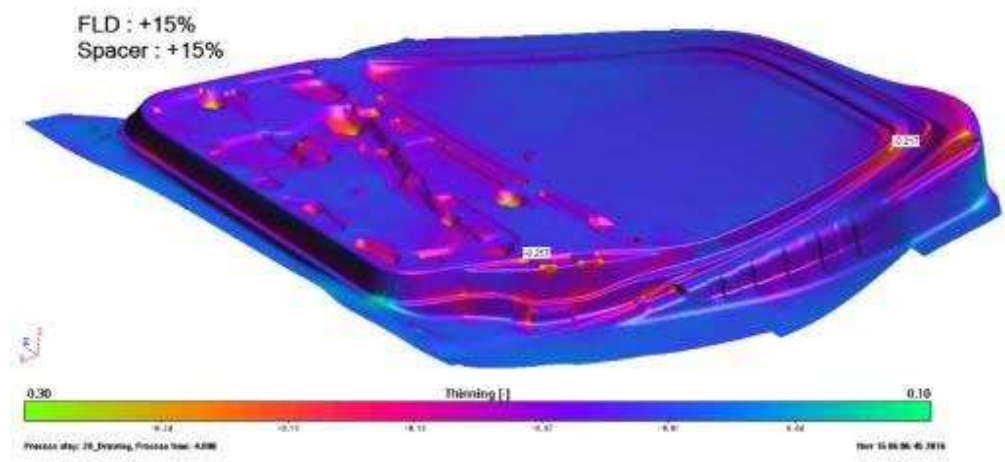

(c)

Fig. 57 Thickness distribution in the final part (a) 15\% decrease in ductility and spacer height, (b) normal condition, and (c) with a 15\% increase in ductility and spacer height

The distribution of surface strains is also shown in Figure (58).

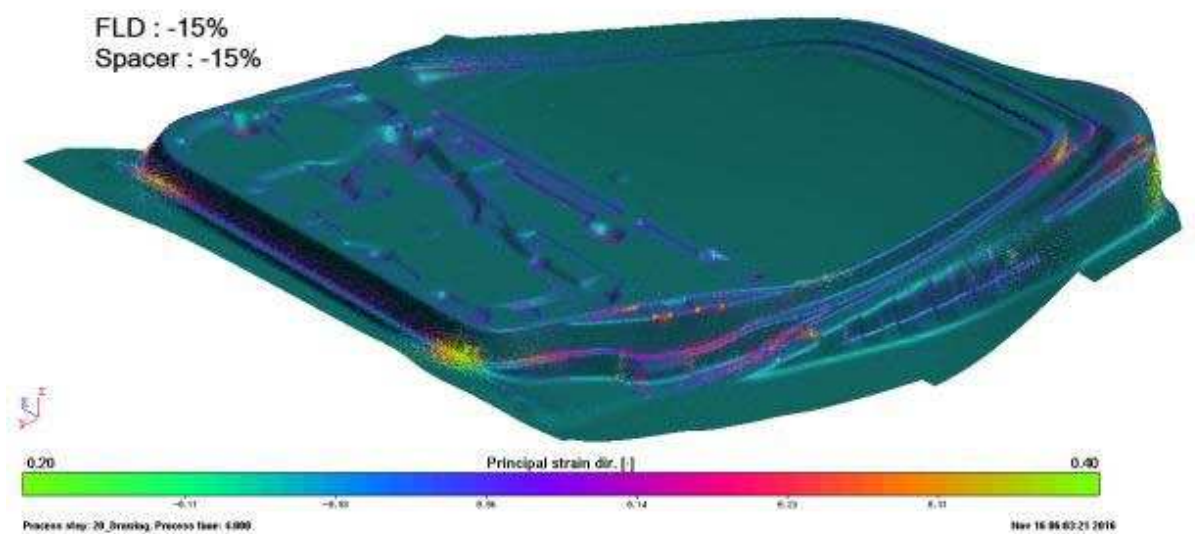

(a) 


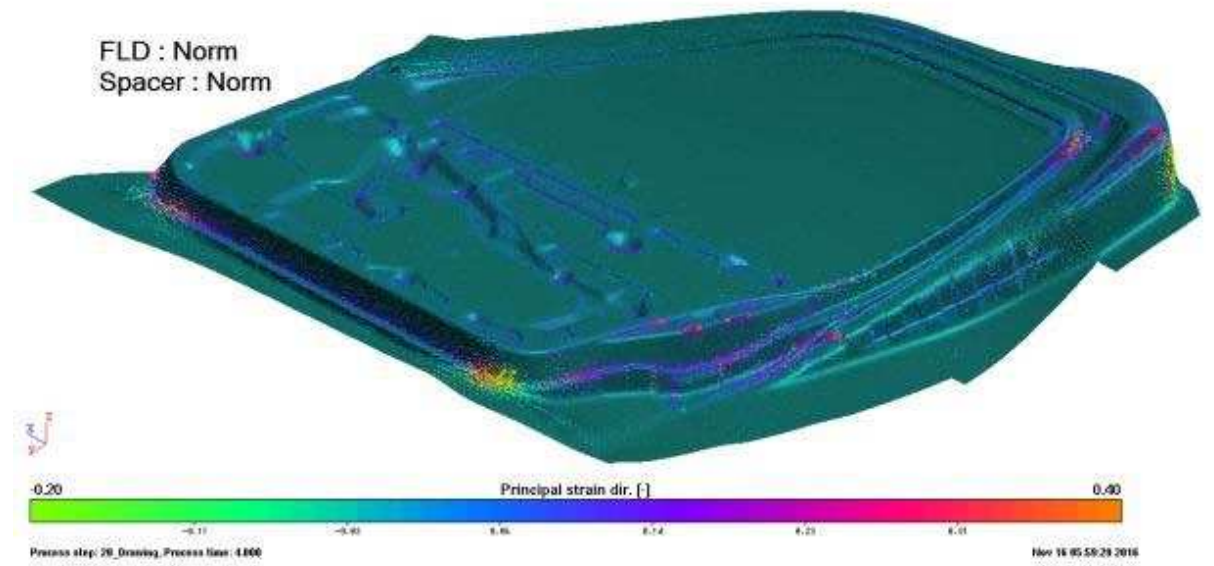

(b)

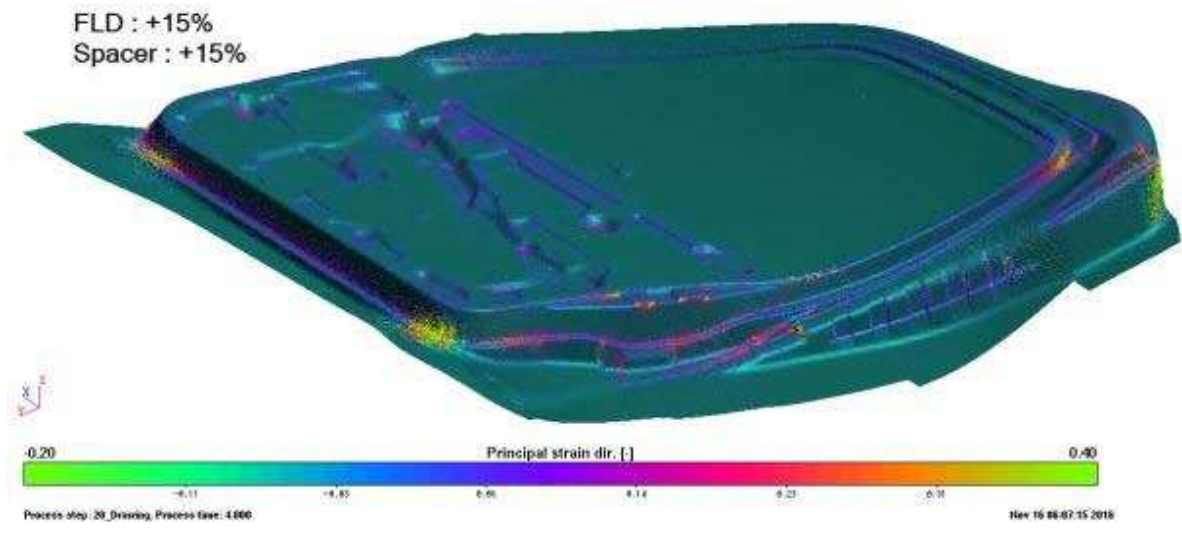

(c)

Fig. 58 Distribute the principal strains (a) with a 15\% decrease in ductility and spacer height, (b) normal state, and (c) with a $15 \%$ increase in ductility and spacer height

Figure (59) shows the trend of changes in thickness distribution and surface strains per three levels of the initial thickness of the sheet (0.6-0.7-0.8 mm). As can be seen, the thinning decreases with increasing thickness. This is the result of the experiments. Also, the fact that the high-risk areas predicted by the software have the highest degree of thinning is evident in these shapes. The percentage of thinning in the two critical areas 3 and 4 is also shown in the figures.

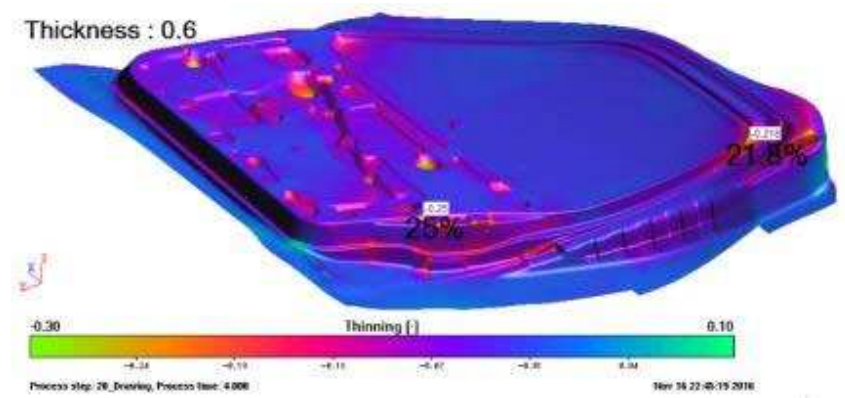

(a) 


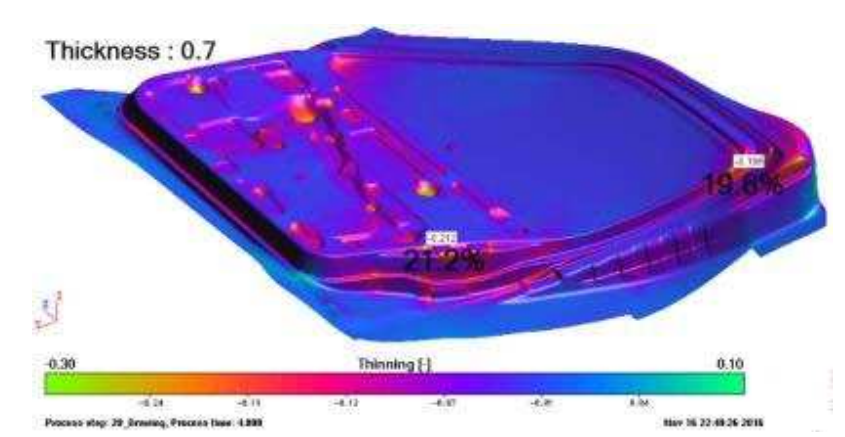

(b)

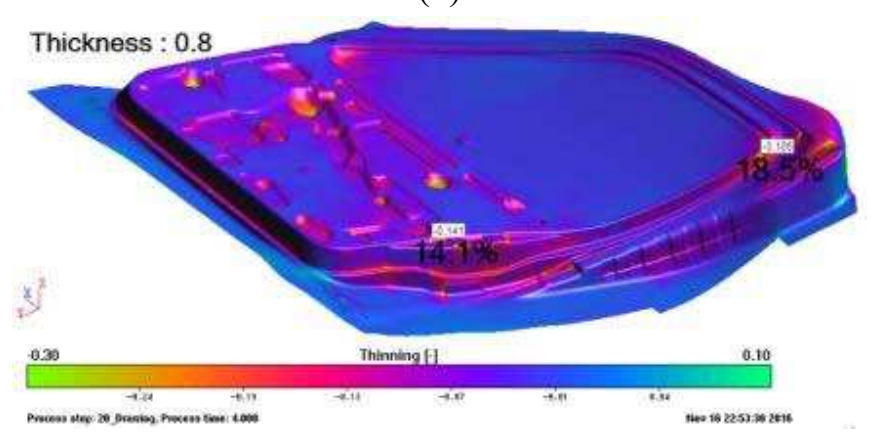

(c)

Fig. 59 Thickness distribution in the tested surfaces (a) 0.6, (b) 0.7, and (c) 0.8 of the sheet thickness

Due to the strain behavior of the part in the figures of this section and the observations made, it is possible to get the results shown in the diagrams.

\section{Conclusion}

This study was conducted to optimize the deep draw process and provide practical solutions to reduce the financial and time cost of mold testing and reduce possible defects in the process on the production molds of the car body. Considering the effects that the changes of each parameter had on the two thickness strain outputs and surface strains, it can be seen that these effects were almost the same in all four regions, and only slight differences were observed in some parameters.

1. Use of lubricant and reduction of friction coefficient can positively affect the DC04 sheet forming process. This effect on the uniform distribution of strains leads to an increase in the tolerable tolerance of the sheet deformation in the FLD diagram.

2. Considering that in the production of parts of the car body, the dominant phase in the production process is expansion, i.e., occurs in the first quarter, increasing the strain rate and if the dominant traction process is deep, reducing the strain rate will be reduced have a positive effect. With the studied model, if the strain rate is reduced, the shaping of the part will be improved. One of the solutions is to reduce the coefficient of friction mentioned in case 1.

3. In addition to the effect that friction has a tolerable degree of molding, it is possible to increase the allowable percentage of thinning and surface strains before choking and tearing by changing the sheet properties and increasing the safe forming range in the FLD diagram. 
4. Increasing the height of spacers due to their role in distributing the press pressure and adapting it to the conditions and geometry of the die in all areas has a positive effect on the formation of the sheet.

5. Considering the almost different effect of the sheet metal force changes in the critical zone 4 and also on the position of the wall zone 1, it is possible to use the increasing sheet metal force mode and compensate the pressure on these areas by adjusting the height of the balancers with more difference.

6. If it is possible to reduce the friction force significantly, the holder force can be increased.

7. Although the choice of a thicker sheet leads to a reduction in the amount of thinning, but considering the weight of the part and costs, this may not be the right choice, while other parameters such as friction can be changed that do not negatively affect the weight of the final product. On the other hand, it helps to form a more uniform part.

8. The factors that control the deep traction process are quite obvious and interact so complexly that it is not possible to explain the process mathematically accurately in simple terms. Therefore, in future research in this field, to accurately determine these interactions, firstly, sufficient accuracy in the simulation process and the proximity of the simulated model to reality, and secondly, if financial and time facilities allow, design and conduct experiments. Full factorial is recommended.

\section{Conflicts of Interest}

The authors declare they have no conflict of interests.

\section{References}

[1] C.-H. Hung, T. Turk, M. H. Sehhat, and M. C. Leu, "Development and experimental study of an automated laser-foil-printing additive manufacturing system," Rapid Prototyp. J., 2022 .

[2] M. H. Sehhat, J. Chandler, and Z. Yates, "A review on ICP powder plasma spheroidization process parameters," Int. J. Refract. Met. Hard Mater., p. 105764, 2021.

[3] M. H. Sehhat, A. T. Sutton, C.-H. Hung, J. W. Newkirk, and M. C. Leu, "Investigation of Mechanical Properties of Parts Fabricated with Gas-and Water-Atomized 304L Stainless Steel Powder in the Laser Powder Bed Fusion Process," JOM, pp. 1-8, 2021.

[4] M. H. Sehhat and A. Mahdianikhotbesara, "Powder spreading in laser-powder bed fusion process," Granul. Matter, vol. 23, no. 4, p. 89, 2021, doi: 10.1007/s10035-021-01162-x.

[5] M. H. Sehhat, B. Behdani, C.-H. Hung, and A. Mahdianikhotbesara, "Development of an Empirical Model on Melt Pool Variation in Laser Foil Printing Additive Manufacturing Process Using Statistical Analysis," Metallogr. Microstruct. Anal., pp. 1-8, 2021. 
[6] T. Turk, C.-H. Hung, M. Hossein Sehhat, and M. C. Leu, "Methods of Automating the Laser-Foil-Printing Additive Manufacturing Process,” 2021.

[7] T. Liu, C. S. Lough, H. Sehhat, J. Huang, E. C. Kinzel, and M. C. Leu, "In-Situ Thermographic Inspection for Laser Powder Bed Fusion,” 2021.

[8] C.-H. Hung, W.-T. Chen, M. H. Sehhat, and M. C. Leu, "The effect of laser welding modes on mechanical properties and microstructure of 304L stainless steel parts fabricated by laser-foil-printing additive manufacturing," Int. J. Adv. Manuf. Technol., vol. 112, no. 3, pp. 867-877, 2021.

[9] M. H. Sehhat, A. Mahdianikhotbesara, and F. Yadegari, "Verification of Stress Transformation in Anisotropic Material Additively Manufactured by Fused Deposition Modeling (FDM)," 2021.

[10] M. H. Sehhat, A. Mahdianikhotbesara, and F. Yadegari, "Impact of Temperature and Material Variation on Mechanical Properties of Parts Fabricated with Fused Deposition Modelling (FDM) Additive Manufacturing," 2021.

[11] M. Sehhat, A. Mahdianikhotbesara, and M. Hadad, "Formability investigation for perforated steel sheets," SAE Int. J. Mater. Manuf., vol. 15, no. 05-15-02-0012, 2021.

[12] D. Banabic, Sheet metal forming processes: constitutive modelling and numerical simulation. Springer Science \& Business Media, 2010.

[13] A. Bayati, D. Rahmatabadi, K. Soltanmohammadi, M. Pahlavani, and R. Hashemi, "Evaluation of forming limit diagrams using Nakazima out-of-plane test and incremental forming process for two-phase magnesium-lithium alloy sheet," J. Brazilian Soc. Mech. Sci. Eng., vol. 43, no. 2, pp. 1-10, 2021.

[14] N. Najafizadeh, M. Rajabi, R. Hashemi, and S. Amini, "A method and apparatus for determination of the ultrasonic-assisted forming limit diagram," Proc. Inst. Mech. Eng. Part C J. Mech. Eng. Sci., p. 09544062211011509, 2021.

[15] B. Mueller et al., "Added value in tooling for sheet metal forming through Additive Manufacturing," in International Conference on Competitive Manufacturing, 2013, pp. 17.

[16] R. Padmanabhan, M. C. Oliveira, J. L. Alves, and L. F. Menezes, "Influence of process parameters on the deep drawing of stainless steel," Finite Elem. Anal. Des., vol. 43, no. 14, pp. 1062-1067, 2007.

[17] E. A. Alisaraei, R. Hashemi, D. Rahmatabadi, and C. Sommitsch, "Experimental study of forming limit diagram and mechanical properties of aluminum foils processed by the accumulative roll bonding," Mater. Res. Express, vol. 7, no. 12, p. 126511, 2020.

[18] Y. Li et al., "A numerical study on chain-die forming of the AHSS U-channel and contrast with roll forming," Int. J. Mech. Sci., vol. 135, pp. 279-293, 2018.

[19] X. Fu, Y. Qiu, and M. Zhao, "Finite Element Analysis of Stamping of Automobile Beam," Comput. Simul. Appl., vol. 1, no. 1, 2018. 
[20] A. Mahdianikhotbesara, M. H. Sehhat, and M. Hadad, "Experimental Study on MicroFriction Stir Welding of Dissimilar Butt Joints Between Al 1050 and Pure Copper," Metallogr. Microstruct. Anal., 2021, doi: 10.1007/s13632-021-00771-5.

[21] M. A. Hussein, A. A. Abbas, and R. A. Abdul-Nabe, "Enhancement the Mechanical Properties of the Deep Drawing Products through Intelligence Design and Finite Element Analysis," Jour Adv Res. Dyn. Control Syst., vol. 10, no. 13, 2018. 